Journal for ImmunoTherapy of Cancer

\title{
Combination of thermally ablative focused ultrasound with gemcitabine controls breast cancer via adaptive immunity
}

\author{
Natasha D Sheybani, ${ }^{1}$ Alexandra R Witter, ${ }^{2}$ Eric A Thim, ${ }^{1}$ Hideo Yagita, ${ }^{3}$ \\ Timothy N J Bullock (D), ${ }^{2}$ Richard J Price (D) ${ }^{1,4}$
}

To cite: Sheybani ND, Witter AR, Thim EA, et al. Combination of thermally ablative focused ultrasound with gemcitabine controls breast cancer via adaptive immunity. Journal for ImmunoTherapy of Cancer 2020;8:e001008. doi:10.1136/ jitc-2020-001008

- Additional material is published online only. To view please visit the journal online (http://dx.doi.org/10.1136/jitc2020-001008).

NDS and ARW contributed equally.

Accepted 22 July 2020

Check for updates

(c) Author(s) (or their employer(s)) 2020. Re-use permitted under CC BY-NC. No commercial re-use. See rights and permissions. Published by BMJ.

${ }^{1}$ Biomedical Engineering, University of Virginia, Charlottesville, Virginia, USA ${ }^{2}$ Pathology, University of Virginia, Charlottesville, Virginia, USA

${ }^{3}$ Department of Immunology, Juntendo University Graduate School of Medicine, Bunkyo-ku, Tokyo, Japan

${ }^{4}$ Radiology \& Medical Imaging, University of Virginia, Charlottesville, Virginia, USA

\section{Correspondence to}

Dr Richard J Price;

rprice@virginia.edu

Dr Timothy N J Bullock; tb5v@virginia.edu

\section{ABSTRACT}

Background Triple-negative breast cancer (TNBC) remains recalcitrant to most targeted therapy approaches. However, recent clinical studies suggest that inducing tumor damage can render TNBC responsive to immunotherapy. We therefore tested a strategy for immune sensitization of murine TNBC (4T1 tumors) through combination of focused ultrasound (FUS) thermal ablation and a chemotherapy, gemcitabine (GEM), known to attenuate myeloid-derived suppressor cells (MDSCs). Methods We applied a sparse-scan thermally ablative FUS regimen at the tumor site in combination with systemically administered GEM. We used flow cytometry analysis to investigate the roles of monotherapy and combinatorial therapy in mediating local and systemic immunity. We also tested this combination in Rag $1^{-/-}$ mice or T cell-depleted wild-type mice to determine the essentiality of adaptive immunity. Further, we layered Programmed cell death protein 1 (PD-1) blockade onto this combination to evaluate its impact on tumor outgrowth and survival.

Results The immune-modulatory effect of FUS monotherapy was insufficient to promote a robust $\mathrm{T}$ cell response against $4 \mathrm{~T} 1$, consistent with the dominant MDSC-driven immunosuppression evident in this model. The combination of FUS+GEM significantly constrained primary TNBC tumor outgrowth and extended overall survival of mice. Tumor control correlated with increased circulating antigen-experienced T cells and was entirely dependent on T cell-mediated immunity. The ability of FUS+GEM to control primary tumor outgrowth was moderately enhanced by either neoadjuvant or adjuvant treatment with anti-PD-1.

Conclusion Thermally ablative FUS in combination with GEM restricts primary tumor outgrowth, improves survival and enhances immunogenicity in a murine metastatic TNBC model. This treatment strategy promises a novel option for potentiating the role of FUS in immunotherapy of metastatic TNBC and is worthy of future clinical evaluation. Trial registration numbers NCT03237572 and NCT04116320.

\section{BACKGROUND}

Metastatic breast cancer (BrCa), particularly the triple-negative breast cancer (TNBC) phenotype, is resistant to most chemical and molecularly targeted therapeutic approaches. Interestingly, TNBC is often infiltrated with immune cells, and the presence of these cells has been shown to have a favorable prognosis in patients treated with neoadjuvant chemotherapy. ${ }^{1}$ Early studies in the use of immunotherapies targeting the PD-1/Programmed death-ligand 1 (PD-L1) checkpoint inhibitory axis showed some efficacy ${ }^{2-4}$ in TNBC compared with other BrCa subtypes, which are generally recalcitrant to checkpoint blockade. Activity in the TNBC subtype may be related to the relatively high immune infiltration and correlated with the higher mutational burden observed in TNBC. Greater immunotherapy efficacy in TNBC has been recently observed with the use of antibodies targeting the PD-1/PD-L1 checkpoint inhibitory axis in combination with Nab-paclitaxel. ${ }^{5}$ This outcome suggests that inducing tumor damage augments antitumor immunity, either by promoting antigen availability or disrupting the immunosuppressive tumor microenvironment (TME) found in TNBC.

Among the potential networks in TNBC that could constrain the activity of antitumor immunity is the presence of immunosuppressive myeloid cell subsets. These have the capacity to impair adaptive immunity and promote tumor growth and metastasis. Among these cell types, myeloid-derived suppressor cells (MDSCs) prevail as a heterogeneous population of immature myeloid cells, which serve the eponymous role of suppressing the antitumor immune response, limiting both $\mathrm{T}$ cell activation and effector functions. ${ }^{67}$ Increased levels of this cell type have been demonstrated in tumor tissues of patients with primary $\mathrm{BrCa}$, while those with metastatic disease bear the highest abundance of circulating MDSCs. ${ }^{8}$ Studies have 
shown that approaches that either stimulate myeloid cells with inflammatory mediators or eliminate MDSC can improve antitumor immunity. ${ }^{9-12}$

To this end, the central premise put forth in this study is that focused ultrasound (FUS) - a safe, noninvasive and nonionizing strategy for localized acoustic energy deposition into tissues-can synergize with immunotherapy in a murine model of metastatic TNBC. FUS is capable of rapidly heating tumors to thermally ablative temperatures. Its extracorporeal application obviates the need for catheterization, injection or implantation. FUS can be targeted with millimeter precision under MRI or ultrasound guidance, thereby allowing for thermal damage and destruction of tumor tissue without compromising healthy intervening or peripheral tissues. The bioeffects of FUS hold distinct implications for tumor antigenicity, immune cell activation and trafficking. ${ }^{13} 14$ Thermally active FUS regimes have elicited antitumor immune responses in implantable models of melanoma, ${ }^{15}$ pancreatic, ${ }^{16}$ prostate, ${ }^{17-19}$ colon, ${ }^{20}$ kidney $^{21}{ }^{22}$ and $\mathrm{BrCa}^{23}$ Pertaining to the challenge of myeloid cell immunosuppression in TNBC, thermally ablative FUS has been shown to induce the expression of heat shock proteins ${ }^{2425}$ and proinflammatory cytokines including interleukin 12 (IL-12), interferon- $\gamma$ (IFN $\gamma$ ) and tumor necrosis factor- $\alpha$ $(\mathrm{TNF} \alpha)$ from a variety of cancer cell lines and after in vivo treatment of tumors. ${ }^{26}{ }^{27}$ Whether the ability of FUS to induce these inflammatory mediators is sufficient to overcome myeloid suppression in the context of $\mathrm{BrCa}$ is currently under debate, with some studies showing activation of antigen-presenting cells and $\mathrm{T}$ cell recruitment in patients with $\mathrm{BrCa}$ treated with thermally ablative FUS, ${ }^{28} 29$ while others show that additional innate stimuli are needed to support antitumor immunity. ${ }^{2330}$ Notably, some studies have suggested that a sparse-scan thermal ablation regimen more effectively recruits and activates dendritic cells (DCs) and antitumor immunity than total thermal ablation, perhaps by limiting thermal denaturation of tumor antigens and innate stimuli. ${ }^{31}$

Based on the improved myeloid cell maturation that occurs with sparse-scan regimens, we herein tested the ability of a sparse-scan, partial thermal ablation FUS regimen as a monotherapy to promote antitumor immunity in an aggressive, syngeneic model of metastatic murine TNBC with extensive granulocytic MDSC involvement that is recalcitrant to anti-PD-1. While some activity is evident with the partial ablation approach, significantly greater control was achieved by targeting MDSC inhibition in combination with thermally ablative FUS. This control was completely dependent on the adaptive immune response.

Moreover, we demonstrate that layering anti-PD-1 immune checkpoint blockade onto this combinatorial regimen moderately improves tumor growth restriction. These data suggest that, in disease settings where myeloid immunosuppression predominates, allied approaches to attenuate myeloid immunosuppression may be employed to reveal the full immunotherapeutic potential of thermally ablative FUS. Once immunosuppressive myeloid cells are accounted for, FUS treatment can promote adaptive immunity that in turn potentiates immune checkpoint blockade.

\section{METHODS}

\section{Cell line maintenance}

4T1 and E0771 cell lines were maintained in RPMI1640 (+L glut) or Dulbecco's Modified Eagle's Medium (DMEM) (4.5 g/L D-glucose, L-glutamine), respectively, supplemented with $10 \%$ Fetal Bovine Serum (FBS) at $37^{\circ} \mathrm{C}$ and $5 \% \mathrm{CO}_{2}$. Thawed cells were cultured for up to three passages and maintained in logarithmic growth phase for all experiments. Cells tested negative for mycoplasma.

Eight-week-old to 12-week-old female BALB/c or C57Bl/6 mice were obtained from NCI Charles River (NCI CRL) or The Jackson Laboratory. Female BALB/c Rag $1^{-/-}$mice were obtained from The Jackson Laboratory. $4 \mathrm{~T} 1$ or E0771 cells $\left(4 \times 10^{5}\right)$ were subcutaneously implanted into the right flank of mice. Mice were housed on a 12-hour/12-hour light/dark cycle and supplied food ad libitum. Tumor outgrowth was monitored via digital caliper measurements. Tumor volume was calculated as follows: volume $=\left(\right.$ length $\times$ width $\left.h^{2}\right) / 2$. Approximately 14 days (4T1) or 21 days (E0771) following tumor implantation, mice were randomized into groups in a manner that ensured matching mean starting tumor volume across experimental groups.

\section{In vivo ultrasound-guided FUS partial thermal ablation}

Mice were treated with FUS either 14 days (4T1 cohorts) or 22 days (E0771) postimplantation. On treatment day, mice were anesthetized with intraperitoneal injection of ketamine (50 mg/kg; Zoetis) and dexdomitor $(0.25 \mathrm{mg} /$ $\mathrm{kg}$; Pfizer) in sterilized $0.9 \%$ saline. Mouse flanks were shaved and depilated, following which ultrasound-guided FUS thermal ablation was performed using one of the two systems. System and treatment details are provided in online supplementary materials and methods. Mice that did not receive FUS treatment consistently underwent anesthesia and depilation of the flank. Additionally, these mice underwent a 'sham' treatment consisting of exposure to the $37^{\circ} \mathrm{C}$ degassed water bath exposure for 6 min. Following 'sham' or FUS treatment, all mice were moved to a heating pad and given Antisedan for anesthesia reversal and recovery.

\section{Gemcitabine therapy}

Gemcitabine (GEM; $1.2 \mathrm{mg}$ /mouse in $500 \mu \mathrm{L}$ volume; Mylan) diluted in $0.9 \%$ saline and filter sterilized through a $0.2 \mu \mathrm{m}$ syringe filter was administered intraperitoneally once a week on the day of FUS treatment, following which administration was repeated for an additional 2 weeks. Administration of GEM doses was based on existing literature demonstrating the use of GEM for inhibition of MDSCs in $4 \mathrm{~T} 1 .^{12}$ The initial dose of GEM was administered immediately prior to 'sham' or FUS treatment. 
Mice that did not receive GEM received an intraperitoneal injection of 'vehicle' treatment $(500 \mu \mathrm{L}$ of sterile $0.9 \%$ saline) at the time points specified.

\section{PD-1 blockade therapy}

For checkpoint inhibitor therapy, the rat anti-mouse PD-1 antibody ( $\alpha$ PD-1, RMP1-14) diluted in sterilized 0.9\% saline was administered intraperitoneally every 3 days for a total of five doses (200 $\mathrm{pg}$ per mouse). Treatment was initiated on day 7 ('early $\alpha$ PD-1') or day 17 ('delayed $\alpha \mathrm{PD}-1$ ').

\section{T cell depletions}

$\mathrm{T}$ cell depletion antibodies-anti-CD8 (2.43 clone; Bio X Cell) and anti-CD4 (GK1.5 clone; Bio X Cell)-were diluted in sterilized $0.9 \%$ saline and administered intraperitoneally every 3 to 4 days starting at day 20 (6 days post-FUS) for a total of seven doses (100 $\mu$ g of each antibody for a total $200 \mu \mathrm{g}$ per mouse).

\section{Immunohistochemistry}

On day 14, sham or FUS-exposed tumors were excised and fixed in $10 \%$ neutral buffered formalin (Sigma). Fixed tumors were paraffin embedded, sectioned and stained for hematoxylin and eosin. Digital images of stained slides were acquired using the Vectra 3.0 Automated Quantitative Pathology Imaging System (Akoya Biosciences). Whole slide screening and image capture were subsequently performed using Phenochart 1.0.8 (Akoya Biosciences).

\section{Flow cytometry}

Mice were bled at days 21 and 28 via tail vein and samples were RBC lysed (Hybri-Max; Sigma) and stained for flow cytometry analysis. At 31 days post-tumor implantation, tissues were obtained from euthanized, tumor-bearing animals for immune response assessment. In order to gain resolution into tissue resident versus vascular immune cell populations, mice were injected intravenously with rat anti-mouse CD45 FITC (clone 30-F11; BD Biosciences) $\sim 3$ min prior to euthanasia. 4T1 tumors, spleens, cardiac blood, axillary and brachial tumor-draining lymph nodes (tumor-DLNs; pooled), and nondraining inguinal lymph nodes were harvested, processed and stained for flow cytometry analysis. Additional details are provided in online supplementary materials and methods.

Samples were acquired on an Attune NxT flow cytometer (ThermoFisher Scientific) and data were analyzed with FlowJo (TreeStar) or FCS Express (De Novo Software). A representative gating strategy for granulocytic myeloid-derived suppressor cell (G-MDSC) and CD44 ${ }^{+} \mathrm{T}$ cells is provided in online supplementary figure 1.

\section{Statistical analysis}

All statistical analyses were performed in GraphPad Prism 8 (GraphPad Software). A detailed description of statistical methods for each experiment is provided in the corresponding figure legend.

\section{Animal study approval}

All animal work was performed under a protocol approved by the Animal Care and Use Committee at the University of Virginia and conformed to the National Institutes of Health guidelines for the use of animals in research.

\section{RESULTS}

Partial thermal ablation of established TNBC tumors promotes peripheral DC activation but has limited impact on the presence of $T$ cells and other myeloid cell subsets

To achieve partial thermal ablation of 4T1 tumors, we used an ultrasound-guided FUS system equipped with a single element therapeutic transducer driven at $3 \mathrm{MHz}$ (figure 1A; online supplementary figure 2). A grid of sonications was overlaid on the ultrasound-visible tumor and ablated in a raster pattern under B-mode ultrasound guidance (figure $1 \mathrm{~B}-\mathrm{C}$ ). The exceptionally small focus of this system rendered a low ablation fraction $(\sim 10 \%-20 \%$ of total tumor volume). Immediately following ablation, tumors displayed evidence of coagulative necrosis in the ablated zone with surrounding periablative margins (figure 1D). One week following FUS partial thermal ablation, tumors and secondary lymphoid organs were excised for immunological characterization by flow cytometry (figure 1B). FUS partial thermal ablation of 4T1 tumors conferred a significant increase ( 2.5-fold) in the absolute number of CD11c-hi DCs within the axillary tumor-draining lymph node (aDLN) of mice (figure 1E). While this was accompanied by a nearly threefold elevation in the absolute number of $\mathrm{CD} 86^{+}$DCs within the aDLN (figure $1 \mathrm{~F}$ ), the percentage of DCs expressing CD86 did not change (figure 1G). Increased numbers of DCs-and CD86 ${ }^{+}$DCs in particular-suggest FUS is promoting the maturation or trafficking of these cells in the DLNs, where they could encounter and activate $\mathrm{T}$ cells. However, this did not translate to tumor growth restriction (data not shown). We also did not observe significant differences in the absolute number of activated $\mathrm{T}$ cells in $4 \mathrm{~T} 1$ tumors (figure $1 \mathrm{H}$ ) or DLNs (data not shown) following FUS exposure, suggesting limitations in the ability of FUS-activated DC to further drive an antitumor $\mathrm{T}$ cell response.

Immune profiling by flow cytometry revealed that, irrespective of FUS exposure, $\sim 75 \%$ of the intratumoral $\mathrm{CD} 45^{+}$immune cell population is comprised of $\mathrm{CD}_{11 \mathrm{~b}^{+}}$ myeloid cells (figure 1I). Similarly, approximately $90 \%$ of the circulating immune cell population in 4T1 tumorbearing mice is comprised of myeloid cells, a striking 3.3fold elevation in circulating myeloid burden compared with naive mice (online supplementary figure 3). Notably, $\mathrm{Ly}_{6 \mathrm{G}}{ }^{+}$granulocytic myeloid-derived suppressor cells (G-MDSCs) significantly dominated the immune cell repertoire within $4 \mathrm{~T} 1$ tumors relative to other myeloid cell subsets, including $\mathrm{F} 4 / 80^{+}$macrophages, Ly6C ${ }^{+}$ monocytic myeloid-derived suppressor cells (M-MDSCs) and CD11c-hi DCs (figure 1J). FUS partial thermal ablation did not significantly alter the absolute number per 


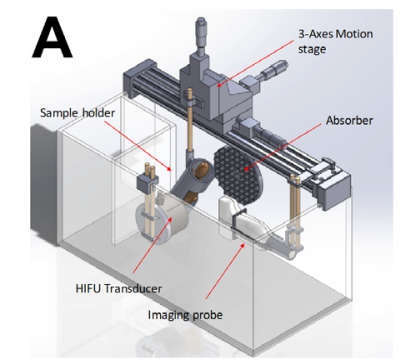

B
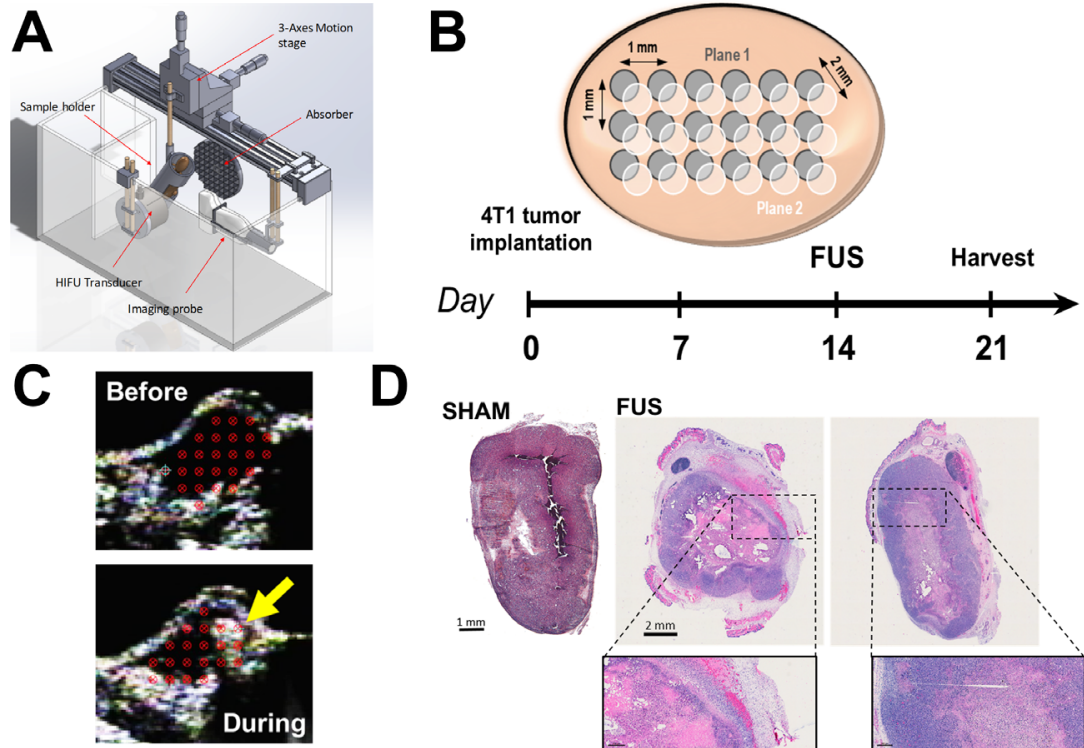

D
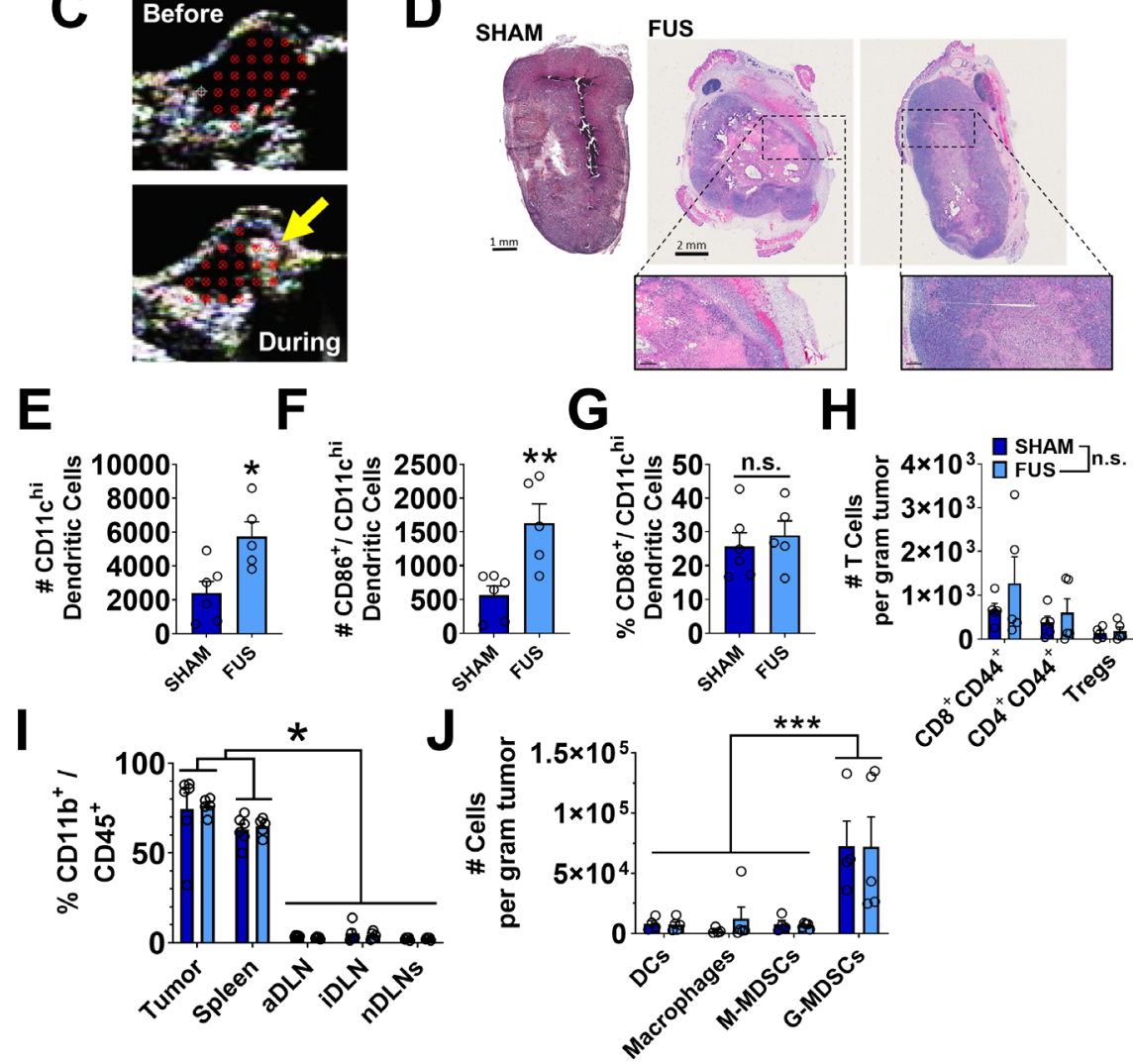

Figure 1 Partial thermal ablation of established TNBC tumors promotes peripheral DC activation but has limited impact on the presence of T cells and other myeloid cell subsets. (A) Design overview of a custom ultrasound-guided FUS system consisting of a $3.3 \mathrm{MHz}$ single-element transducer orthogonally co-registered to an $8 \mathrm{MHz}$ linear ultrasound imaging array. The tumor-bearing flank of each anesthetized mouse was acoustically coupled to ultrasound transducers via degassed water bath maintained at $37^{\circ} \mathrm{C}$. 'Sham' mice were similarly positioned but did not undergo sonications. (B) Schematic illustration of FUS partial thermal ablation scheme and study layout for evaluation of immune sequelae in 4T1 tumor-bearing mice. A grid of sonications was applied in a raster pattern onto the B-mode ultrasound-visible tumor. In total, two planes of sonication spaced $2 \mathrm{~mm}$ apart were applied to each tumor. Grid points were spaced $1 \mathrm{~mm}$ apart within a single plane. One week following thermal ablation, tumors and secondary lymphoid organs were excised for sham $(n=6)$ or FUS-treated $(n=5)$ mice and processed for flow cytometry. (C) Representative B-mode ultrasound images of ectopic 4T1 tumors either before (top) or during (bottom) FUS exposure. Sonication grid depicting targets (red points) is superimposed on B-mode image during treatment. Subsequent to thermal ablation, hyperechoic signatures (yellow arrow) are occasionally observed. (D) Representative H\&E staining of either sham 4T1 tumors or those resected immediately following FUS partial thermal ablation. Zoomed insets depict the transition from necrotic to intact tumor tissue within the periablative zone (scale bars $=400 \mu \mathrm{m}$ and $300 \mu \mathrm{m}$ on left and right inset, respectively). (E) Absolute number of CD11c-hi DCs in the axillary tumor-draining lymph node (aDLN) of $4 T 1$ tumor-bearing mice. ${ }^{*} \mathrm{p}=0.0136$ vs sham. (F) Absolute number of CD86 ${ }^{+} \mathrm{CD} 11 \mathrm{c}-\mathrm{hi}$ DCs in the aDLN. ${ }^{* *} \mathrm{p}=0.0063$ vs sham. (G) Percentage of $\mathrm{CD}^{+} 6^{+}$subset out of total CD11c-hi DCs within aDLN. (H) Absolute number of intratumoral CD $44^{+} \mathrm{CD} 8^{+}$and $\mathrm{CD} 44^{+} \mathrm{CD} 4^{+} \mathrm{T}$ cells and regulatory $T$ cells (Tregs) per gram tumor. (I) Percentage of $\mathrm{CD} 11 \mathrm{~b}^{+}$myeloid cells out of total $\mathrm{CD} 45^{+}$immune cells across tumor, spleen, aDLN, inguinal DLN (iDLN), and nontumor draining axillary and inguinal $L N s$ ( $(n D L N s)$. ${ }^{*} \mathrm{p}<0.05$ vs all other groups (irrespective of FUS exposure; specifically, tumor vs spleen: $p=0.0226$; tumor, spleen vs all other organs: $p<0.0001$ ). (J) Absolute number of intratumoral myeloid cells (CD11C-hi DCs, F4/80 macrophages, Ly6 $\mathrm{C}^{+}$monocytic myeloid-derived suppressor cells (M-MDSCs), Ly6G+ granulocytic myeloid-derived suppressor cells (G-MDSCs)) per gram 4T1 tumor. ${ }^{* * *} \mathrm{p}=0.0001$ vs all other cell types (irrespective of FUS exposure). All data represented as mean \pm SEM. Significance assessed by unpaired t-test $(\mathrm{F}-\mathrm{H})$ or two-way analysis of variance followed by Tukey multiple comparison correction (I-K). 'n.s.'=not significant. DCs, dendritic cells; FUS, focused ultrasound; HIFU, high-intensityfocused ultrasound. 
gram tumor of these myeloid cell subsets. These observations led us to formulate the hypothesis that widespread immunosuppressive mechanisms associated with the 4T1 TME must be addressed in order to facilitate the $\mathrm{T}$ cell response to FUS.

\section{FUS partial thermal ablation in combination with GEM constrains primary TNBC tumor outgrowth and extends overall survival}

Our observation of the overwhelming MDSC burden following 4T1 tumor implantation warranted implementation of an allied therapeutic strategy in order to counter this immunosuppressive barrier. To this end, we tested a combinatorial paradigm incorporating GEM, a myelosuppressive chemotherapy demonstrated to inhibit MDSCs transiently in the $4 \mathrm{~T} 1$ model (without consequence to $\mathrm{T}$ cell phenotype or function). ${ }^{12}$

To evaluate the efficacy of FUS and GEM in combination, we used a preclinical ultrasound-guided FUS system to achieve partial thermal ablation of established 4T1 tumors $14 \mathrm{~d}$ after tumor implantation (average tumor volume of $\sim 100 \mathrm{~mm}^{3}$ ). In combination with the single session of FUS thermal ablation, we initiated GEM therapy $(1.2 \mathrm{mg} / \mathrm{mouse})$, which was then readministered weekly for a total of three GEM doses (figure 2A). Combinatorial therapy synergized to produce significant constraint of 4T1 tumor outgrowth compared with sham and monotherapy groups (figure $2 \mathrm{~B}-\mathrm{C}$ ).

By termination of treatments at day 28, 4T1 tumors exposed to FUS+GEM combination saw nearly $4 \times$ and $2 \times$ reductions in average volume compared with sham or GEM-exposed tumors, respectively (figure 2B). Twodimensional tumor projections at day 31 postimplantation saw a nearly 2.4-fold reduction in area from sham to combinatorial therapy setting (figure 2D-E). In a fraction of mice $(2 / 10)$ treated with FUS+GEM, we observed complete regression of $4 \mathrm{~T} 1$ tumors, although transient (figure 2C); tumor outgrowth eventually rebounded after termination of treatment(s). 4T1 tumor-bearing mice receiving FUS+GEM treatment additionally saw the greatest extension in overall survival with $\sim 51 \%$ and $\sim 15 \%$ increases in median survival time compared with sham and GEM groups, respectively (HRs: 0.2367 and 0.3352 for FUS+GEM relative to sham and GEM groups, respectively) (figure $2 \mathrm{~F}$ ). We additionally observed that FUS+GEM significantly constrained outgrowth in a separate $\mathrm{C} 57 \mathrm{Bl} / 6$ metastatic mammary carcinoma model, E0771 (online supplementary figure 4).

To further the clinical relevancy of these findings, we applied this combinatorial strategy with the researchgrade analog of a clinical ultrasound-guided FUS system (Theraclion Echopulse) that is already CE marked for applications in breast fibroadenoma, thyroid/parathyroid gland and varicose vein ablation and currently in use for multiple clinical trials leveraging FUS thermal ablation in combination with cancer immunotherapy. We observed that partial thermal ablation using the Theraclion visualization and treatment unit (3 MHz) in combination with GEM controlled 4T1 tumor outgrowth to a degree comparable with that observed with the custom in-house system (online supplementary figure 5). These findings lend credence to the notion that the impact of combining GEM with FUS may be conserved across partial thermal ablation regimens. Moreover, they demonstrate that the efficacy of FUS partial thermal ablation in combination with GEM can be recapitulated on a system with a larger focus and in-line image guidance that is currently in use clinically.

\section{Combination of FUS partial thermal ablation with GEM increases the levels of circulating $T$ cells}

Lymphocytes-in particular CD8 and CD4 T cells-play an important role in responding to tumor antigen and generating a durable antitumor response. Based on the extended protective effect observed in mice treated with FUS+GEM, flow cytometry analysis was performed to evaluate the contribution of T cells in generating systemic and local tumor control. We sampled the circulating immune cell repertoire in 4T1 tumor-bearing mice via serial tail bleeds (days 21 and 28, prior to readministration of GEM) and a terminal cardiac bleed at the time of spleen harvest (day 31) (figure 3A). Combinatorial therapy significantly elevated absolute number of CD8 and CD4 T cells in the circulation at days 21 and 28 (figure 3B-C and $\mathrm{E}-\mathrm{F})$. Moreover, a trend (threefold to fivefold increase) in circulating $\mathrm{T}$ cells was noted in the FUS group relative to sham (figure 3B-C and E-F). From days 21 to 28, systemic CD44-expressing (antigen-experienced) $\mathrm{T}$ cell populations (both CD8 and CD4) saw a steady, significant increase after combinatorial therapy (figure $3 \mathrm{D}$ and $\mathrm{G}$ ). A similar modest trend was noted for the FUS monotherapy group relative to sham and GEM (figure 3D and G). These changes were concordant with a decrease in circulating myeloid $\left(\mathrm{CD} 11 \mathrm{~b}^{+}\right)$cells in GEM-recipient groups, demonstrating the ability of GEM to partially alleviate circulating myeloid burden (figure $3 \mathrm{H}$ ).

Splenomegaly is a common signature that arises in parallel with the leukemoid reaction to $4 \mathrm{~T} 1$ tumors, that is, the expansion of immunosuppressive myeloid cells during tumor progression. We observed that combinatorial therapy most significantly reverses splenomegaly (online supplementary figure 6A-B). Consistent with this observation, immunological characterization of spleens revealed a significant decrease in $\mathrm{CD}_{11} \mathrm{~b}^{+}$myeloid cells-a 24\%-46\% reduction in FUS+GEM spleens relative to sham or monotherapy (figure 3I). While there appeared to be a trend toward more $\mathrm{CD} 11 \mathrm{~b}^{+}$cells in the monotherapy groups compared with the sham, this difference was not significant and there was no difference between these groups in terms of absolute $\mathrm{CD} 11 \mathrm{~b}^{+}$cell numbers within the spleen (data not shown). The decrease in myeloid cells in the combination treatment group was accompanied by a significant corresponding elevation in lymphocytes in the spleen following FUS+GEM treatment. Relative to these sham and GEM groups, combination therapy elevated splenic $\mathrm{CD}^{+} \mathrm{T}$ lymphocytes by 14.5 -fold and 


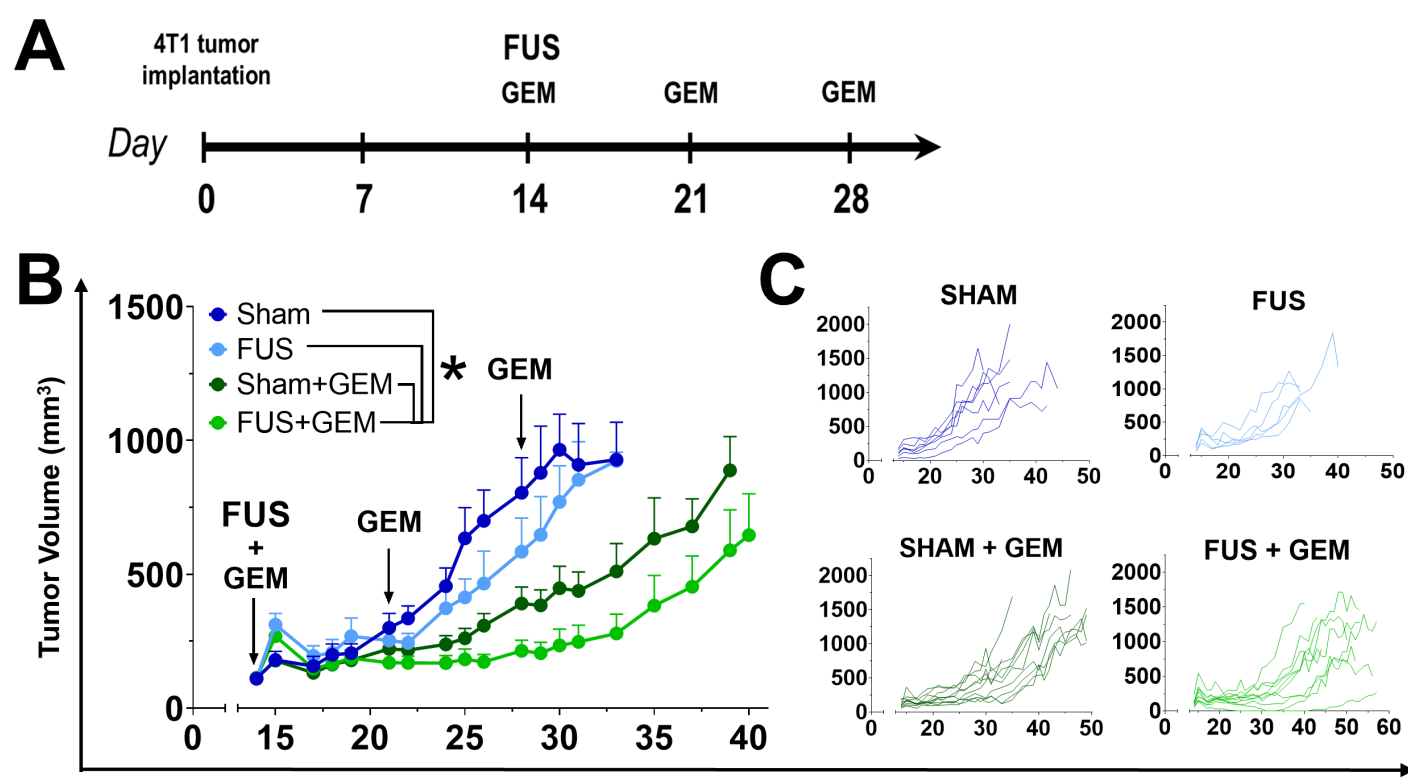

Days Postimplantation
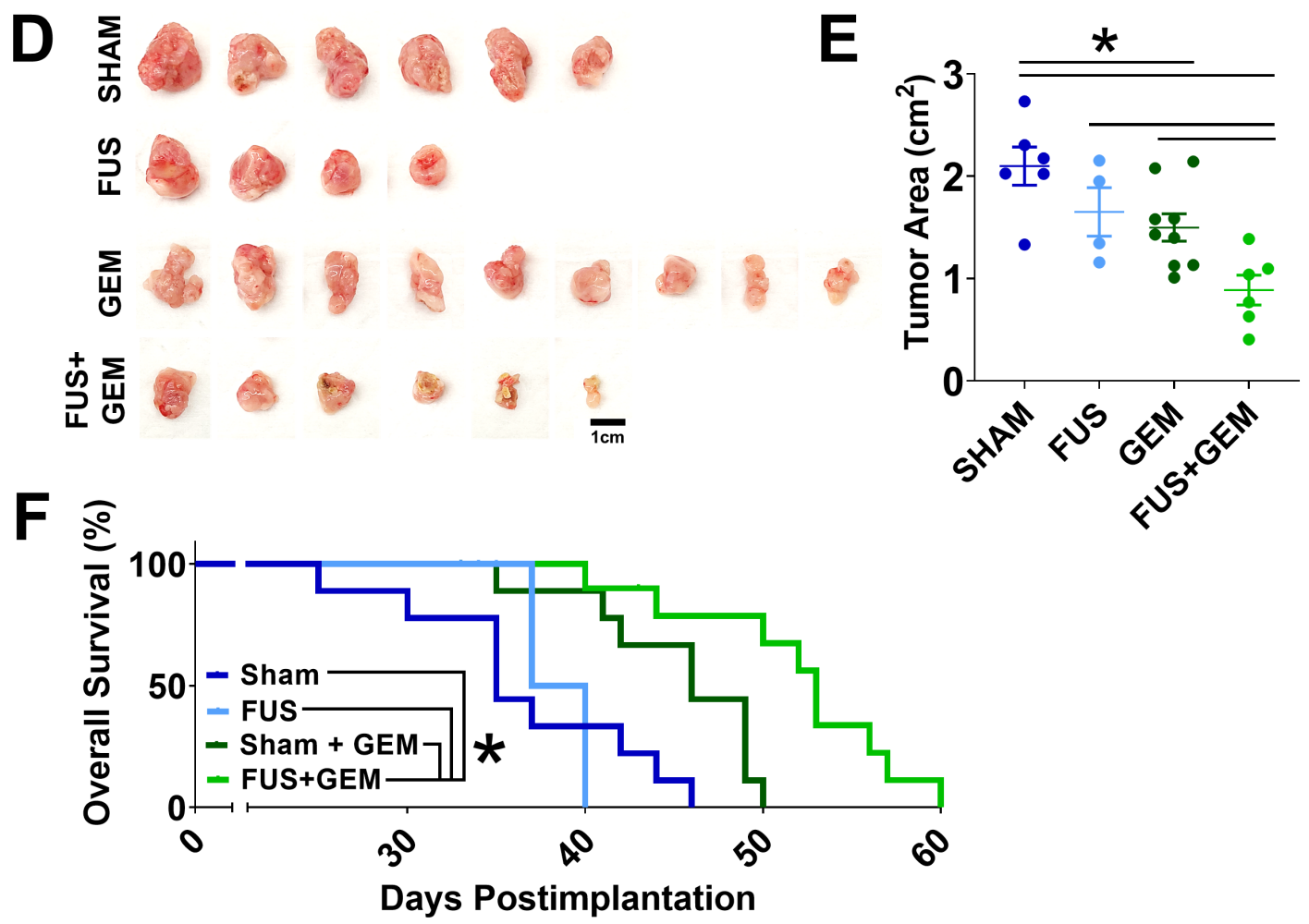

Figure 2 Combination of focused ultrasound (FUS) partial thermal ablation with gemcitabine (GEM) constrains primary triplenegative breast cancer outgrowth and extends overall survival. (A) Overview of experimental design for evaluation combination of FUS with serial GEM treatment in murine mammary carcinoma. (B) Average 4T1 tumor outgrowth in sham ( $n=7$ ), FUS monotherapy $(n=5)$, GEM monotherapy $(n=10)$, and combinatorial FUS+GEM therapy groups $(n=10)$. Data are represented up to select time points corresponding with mouse dropout due to humane endpoints. All data represented as mean \pm SEM. Significance assessed on outgrowth up to day 40 by repeated measures mixed-effects model implementing restricted maximum likelihood method, followed by Tukey multiple comparison correction. ${ }^{*} \mathrm{p}<0.05$ vs all other groups (specifically, sham vs FUS+GEM: $p<0.0001$; FUS vs FUS+GEM: $p<0.0001$; sham+GEM vs FUS+GEM: $p=0.0026)$. (C) $4 T 1$ tumor outgrowth from individual mice in sham, FUS, sham+GEM, or FUS+GEM groups. Data represent outgrowth from initiation of treatment(s) at day 14 up to removal of mouse from study for meeting a humane endpoint. (D) Representative images of 4 T1 tumors excised at day 31. Scale bar=1 cm. (E) Quantification of 2D tumor areas from images in previous panel. (F) Kaplan-Meier curve depicting overall survival of sham treatment $(n=9)$, FUS monotherapy $(n=6)$, GEM monotherapy $(n=10)$, and combinatorial FUS+GEM therapy $(n=10)$ recipient mice. Significance assessed by log-rank (Mantel-Cox) test. * $p<0.05$ vs all other groups (specifically, sham vs FUS: $p=0.2154$; sham vs FUS+GEM: $p<0.0001$; sham vs sham+GEM: $p=0.0050 ; F U S$ vs FUS+GEM: $p=0.0021$; FUS vs sham+GEM: $p=0.0312$; FUS+GEM vs sham+GEM: $p=0.0041)$. 

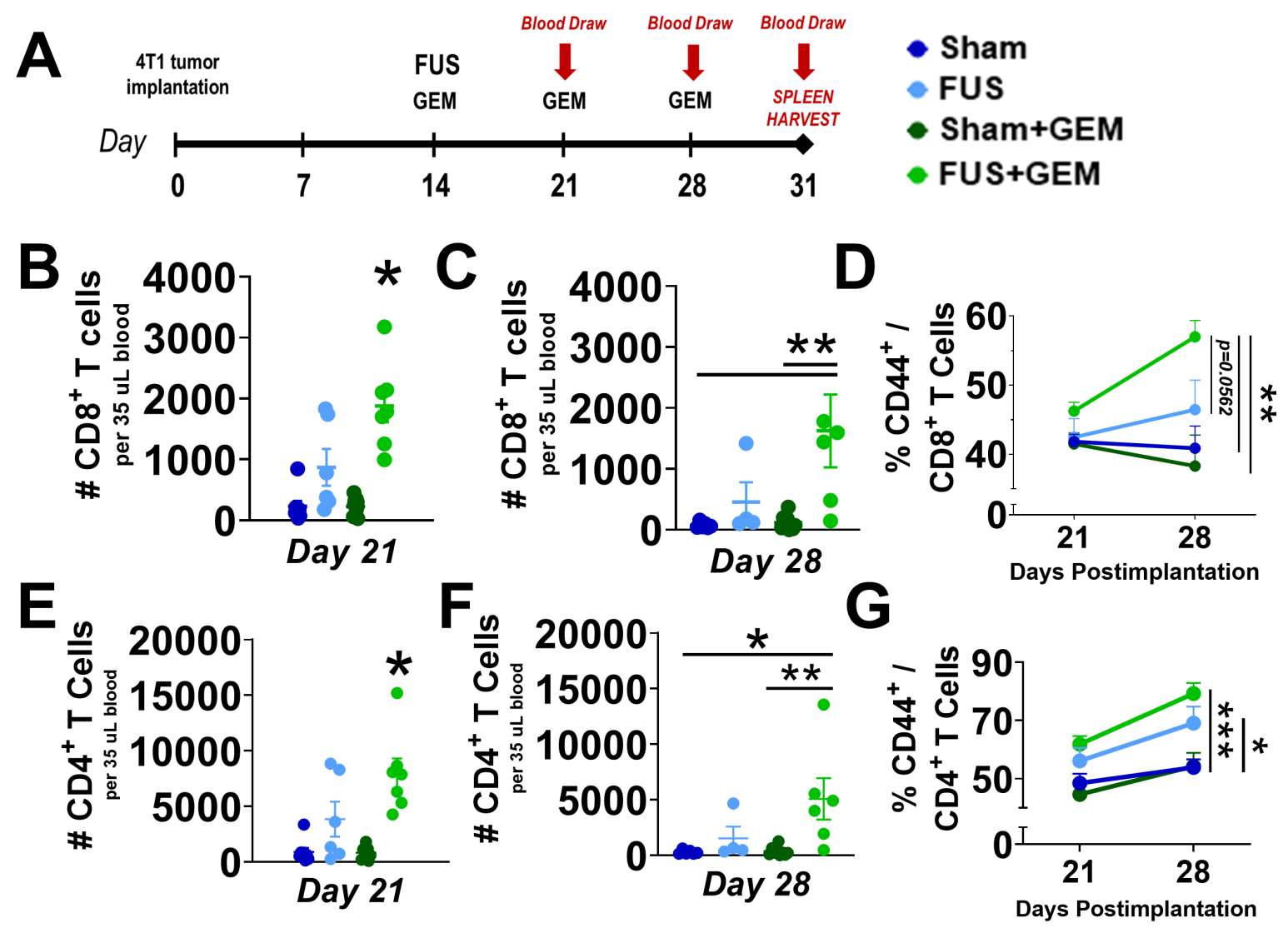

H
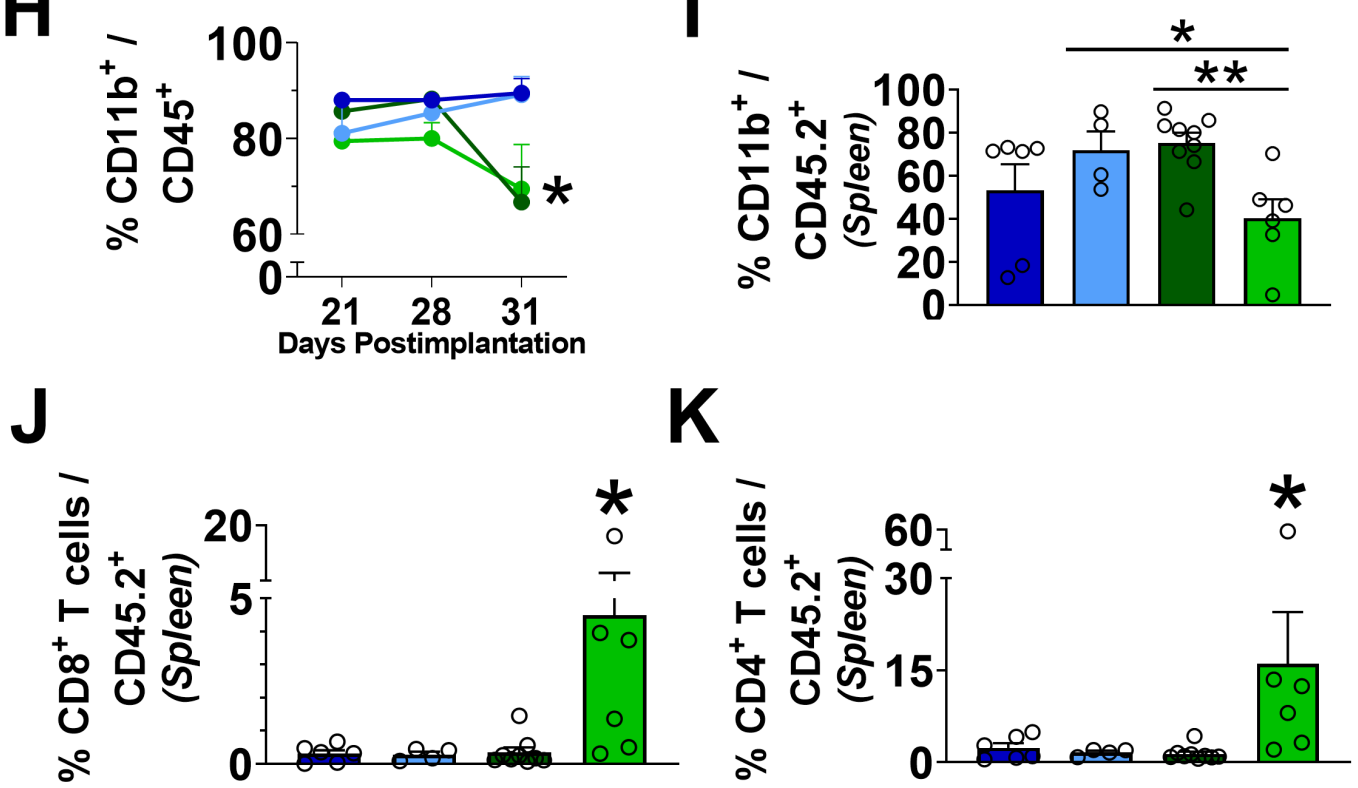

Figure 3 Combination of focused ultrasound (FUS) partial thermal ablation with gemcitabine (GEM) increases the levels of circulating T cells. (A) Overview of experimental design to understand the impact of FUS and/or GEM treatment on circulating immune cells. (B-C) Absolute number of circulating CD8 ${ }^{+} T$ cells at day 21 (B) and day 28 (C). (D) Percentage of circulating $\mathrm{CD}^{+} \mathrm{T}$ cells expressing CD44 from days 21 to 28 . (E-F) Absolute number of circulating CD4 ${ }^{+} \mathrm{T}$ cells at day 21 (E) and day 28 (F). (G) Percentage of circulating CD4 ${ }^{+} \mathrm{T}$ cells expressing CD44 from days 21 to 28 . (H) Percentage of CD11 $\mathrm{b}^{+}$myeloid cells out of total CD45 $5^{+}$immune cell in circulation from days 21 to 31. (I-K) Percentage of myeloid cells (I), CD8 ${ }^{+} \mathrm{T}$ cells $(\mathrm{J})$ and $\mathrm{CD} 4^{+} \mathrm{T}$ cells $(K)$ out of total CD45.2 ${ }^{+}$immune cells. All data represented as mean \pm SEM. All data representative of sham $(n=6-8)$, FUS monotherapy $(n=4-6)$, GEM monotherapy $(n=9)$, and combinatorial FUS+GEM therapy $(n=6-7)$ groups. Significance assessed by analysis of variance followed by Tukey multiple comparison correction (for B, C, E, F) or Fisher's least significant difference (LSD) without multiple comparisons correction (for I-K). Significance (for D, G and H) assessed by repeated measures mixedeffects model implementing restricted maximum likelihood method, followed by Fisher's LSD without multiple comparisons correction. ${ }^{\star} \mathrm{p}<0.05$ vs all other groups unless otherwise indicated. ${ }^{* \star} \mathrm{p}<0.01,{ }^{\star \star \star} \mathrm{p}<0.001$ vs group(s) indicated. 
12.9-fold (figure 3J) and $\mathrm{CD} 4^{+} \mathrm{T}$ lymphocytes by 6.9-fold and 11.6-fold (figure 3K). These elevations were accompanied by a modest increase in percentage of $\mathrm{Foxp}^{+}{ }^{+}$regulatory T cells (Tregs) (online supplementary figure 6E). Additionally, increases in percentage of NK and B cells were noted ( twofold to fivefold) (online supplementary figure $6 \mathrm{C}-\mathrm{D}$ ). These findings indicate that combinatorial therapy with FUS+GEM promotes a systemic lymphocyte response that is discrete from the effects of either intervention alone, which may account for reduced mortality associated with pulmonary metastases.

\section{Combinatorial FUS+GEM therapy does not promote robust local antitumor $T$ cell responses}

Given the robust systemic immune signatures within the blood and spleen following FUS+GEM, we assayed $4 \mathrm{~T} 1$ tumors at a time point within the window of tumor growth restriction and subsequent to termination of treatments (ie, day 31) to interrogate whether tumor control correlates with an increase in the effector functions of the intratumoral $\mathrm{T}$ cell response (figure $4 \mathrm{~A}$ ). Approximately 6 hours prior to euthanasia, mice received intravenous brefeldin A injection to inhibit cytokine secretion for subsequent intracellular cytokine staining by flow cytometry. Immune characterization of tumors at 31 days postimplantation-that is, 3 days subsequent to final GEM administration-revealed no significant changes in absolute number of antigen-experienced $\left(\mathrm{CD} 44^{+}\right)$ $\mathrm{CD}^{+}$or $\mathrm{CD}^{+} \mathrm{T}$ lymphocytes (figure $4 \mathrm{~B}-\mathrm{C}$ ). Moreover, the polyfunctionality of these $\mathrm{T}$ cells, as denoted by IFN $\gamma$ and granzyme B expression, was not significantly altered (figure 4D-E). However, intratumoral functional changes were noted in the myeloid compartment. GEM monotherapy modestly increased IL- $12 \mathrm{p} 40$ production by DCs ( 1.7-fold), but this was not conserved in the combinatorial therapy group (figure $4 \mathrm{~F}$ ). Moreover, while FUS monotherapy generated a trend in elevated TNF $\alpha$ production by intratumoral G-MDSCs, GEM-recipient groups saw a significant increase ( threefold relative to sham) (figure 4G). These findings indicate that changes in the myeloid compartment in response to monotherapy and combination therapy may contribute to tumor control, but are unlikely to drive the protective response entirely. Interestingly, intratumoral $\mathrm{T}$ cell representation correlates poorly with circulating lymphocytes, suggesting a transitory immune response that either cannot be fully characterized at this time point or is hampered by additional modes of immunosuppression.

\section{Protection conferred by combination of FUS and GEM is dependent on adaptive immunity}

Since our findings revealed no obvious advantage or function of adaptive immunity in the local TME, we next investigated the overarching role of the adaptive immune system in protection offered by combinatorial therapy with FUS+GEM. To this end, we utilized an Rag $1^{-/-}$model that is deficient in $\mathrm{T}$ and $\mathrm{B}$ cells to address the hypothesis that mature $\mathrm{T}$ and/or B cells play a role in the observed response. Wild-type (WT) or Rag1 $1^{-/-}$mice-bearing $4 \mathrm{~T} 1$ tumors were randomized into groups in a manner that preserved similarity in average initial tumor volumes. Mice were subsequently treated with either GEM monotherapy or the combination of FUS+GEM. The tumor growth inhibition offered by FUS+GEM was entirely lost in $\mathrm{Rag}^{-/-}$mice relative to their WT counterparts, with average 4T1 tumor volume in Rag $1^{-/-}$mice being over fivefold higher than that of WT mice on termination of treatments (figure 5A). Of note, despite a trend toward loss of protection in $\mathrm{Rag}^{-/-}$mice, tumor outgrowth in response to GEM monotherapy did not significantly stratify between WT and $\mathrm{Rag}^{-/-}$settings (figure 5A). We also observed a complete loss of FUS+GEM-mediated survival benefit over GEM monotherapy in the Rag $1^{-/-}$ setting (figure 5B). While these results demonstrate that an intact adaptive immune response is required for both the overall survival benefit and restriction of primary tumor offered by FUS+GEM therapy, they do not delineate the relative roles of $\mathrm{T}$ and/or $\mathrm{B}$ cells.

Thus, to address the hypothesis that the protective effect of FUS+GEM is specifically dependent on $\mathrm{CD} 4^{+}$and $\mathrm{CD} 8^{+}$ $\mathrm{T}$ cells, we depleted these populations via serial coinjections of CD8-depleting and CD4-depleting antibodies in 4T1 tumor-bearing WT mice on a FUS+GEM background (figure 5C). Depletions were maintained between day 20 and day 39, and flow cytometry analysis of circulating immune cells at day 27 confirmed that the target $\mathrm{T}$ cell populations were effectively depleted in all mice (online supplementary figure 7). Consistent with the tumor escape observed in Rag $1^{-/-}$mice, the protective effect of FUS+GEM on primary tumor outgrowth was diminished in mice receiving $\mathrm{T}$ cell depletion (figure 5D). Average 4T1 tumor volume at termination of depletion maintenance (day 39) was over 2.5-fold higher in T cell-depleted mice. Moreover, a significant diminution in overall survival was observed in the setting of $\mathrm{T}$ cell depletion, with an $~ 23 \%$ reduction in median survival time resulting from $\mathrm{T}$ cell depletion in FUS+GEM-recipient mice (figure 5E). These findings elaborate on our observations in $\operatorname{Rag} 1^{-/-}$mice by indicating a critical role of $\mathrm{CD}^{+}$and $\mathrm{CD}^{+}{ }^{+} \mathrm{T}$ cells in the durable control of $4 \mathrm{~T} 1$ tumors by combinatory therapy with FUS+GEM.

\section{PD-1 blockade therapy moderately improves growth restriction conferred by FUS+GEM}

We observed minimal changes to intratumoral $\mathrm{T}$ cell repertoire and function despite the evident role of adaptive immunity in FUS+GEM therapy. Thus, we postulated that additional mechanisms of intratumoral immunosuppression may be hindering durable immunological control of 4T1 tumors. We investigated the PD-1/PD-L1 axis, a well-established immune checkpoint pathway. Immunological profiling of 4T1 tumors by flow cytometry at day 31 revealed a high percentage of intratumoral $\mathrm{CD}^{+} \mathrm{CD} 44^{+} \mathrm{T}$ cells expressing PD-1 (figure $6 \mathrm{~A}-\mathrm{B}$ ), as well as high mean fluorescence intensity (MFI) of PD-1 on $\mathrm{CD}^{+} \mathrm{CD}^{+} 4^{+} \mathrm{T}$ cells (figure 6C). Aside from a moderate 
A 4T1 tumor

implantation

Day

FUS

\section{GEM}

GEM

HARVEST

- Sham

FUS

Sham+GEM

$0 \quad 7$

14

21

28

31

FUS+GEM

B
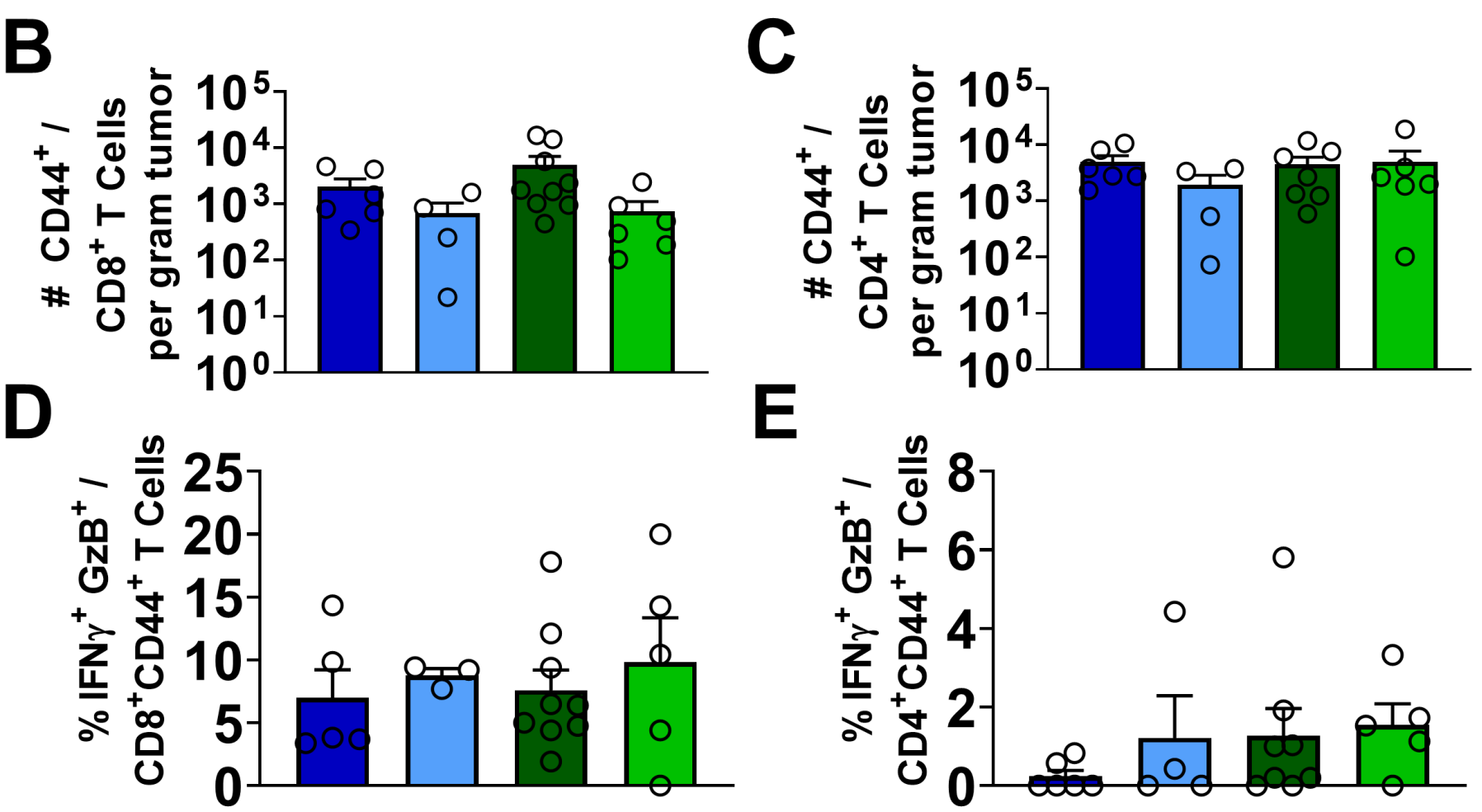

E
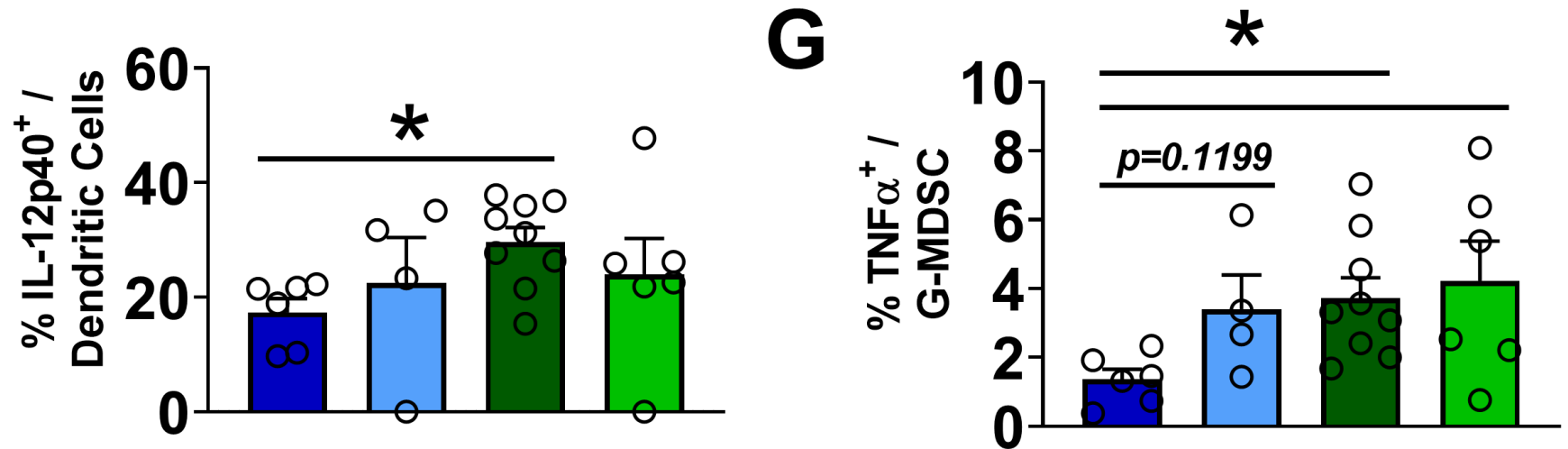

Figure 4 Combinatorial FUS+GEM therapy does not promote robust local antitumor T cell responses. (A) Schematic of experimental design for analysis of immune milieu in tumors and secondary lymphoid organs following FUS and/or GEM treatment. (B, C) Absolute number per gram tumor of $\mathrm{CD}^{+}(\mathrm{B})$ or $\mathrm{CD}^{+}(\mathrm{C}) \mathrm{T}$ cells expressing CD44. (D, E) Percentage of intratumoral $\mathrm{CD} 8^{+} \mathrm{CD} 44^{+}(\mathrm{D})$ or $\mathrm{CD} 4^{+} \mathrm{CD} 44^{+}(\mathrm{E}) \mathrm{T}$ cells dually expressing granzyme $\mathrm{B}(\mathrm{GzB})$ and interferon- $\gamma(\mathrm{IFN} \gamma)$. (F) Percentage of intratumoral DCs expressing IL-12p40. (G) Percentage of intratumoral granulocytic myeloid-derived suppressor cells (G-MDSCs) expressing TNF $\alpha$. Groups not significantly different in (B-E). All data represented as mean $\pm S E M$. All data representative of sham $(n=6)$, FUS monotherapy $(n=4)$, GEM monotherapy $(n=9)$, and combinatorial FUS+GEM therapy $(n=6)$ groups. Significance assessed by analysis of variance followed by Fisher's leastsignificant difference without multiple comparisons correction for all panels. ${ }^{*} \mathrm{p}<0.05$ vs indicated group(s). FUS, focusedultrasound; GEM, gemcitabine; IL, interleukin; TNF $\alpha$, tumor necrosis factor- $\alpha$. 


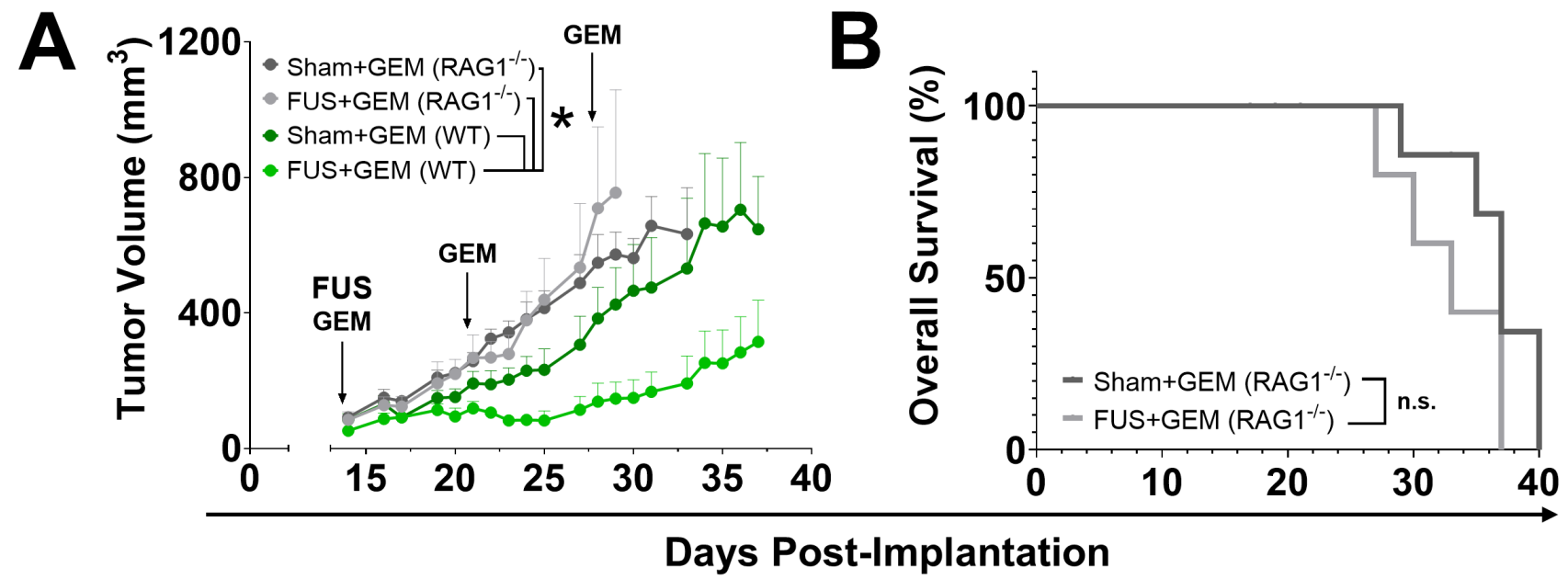

C

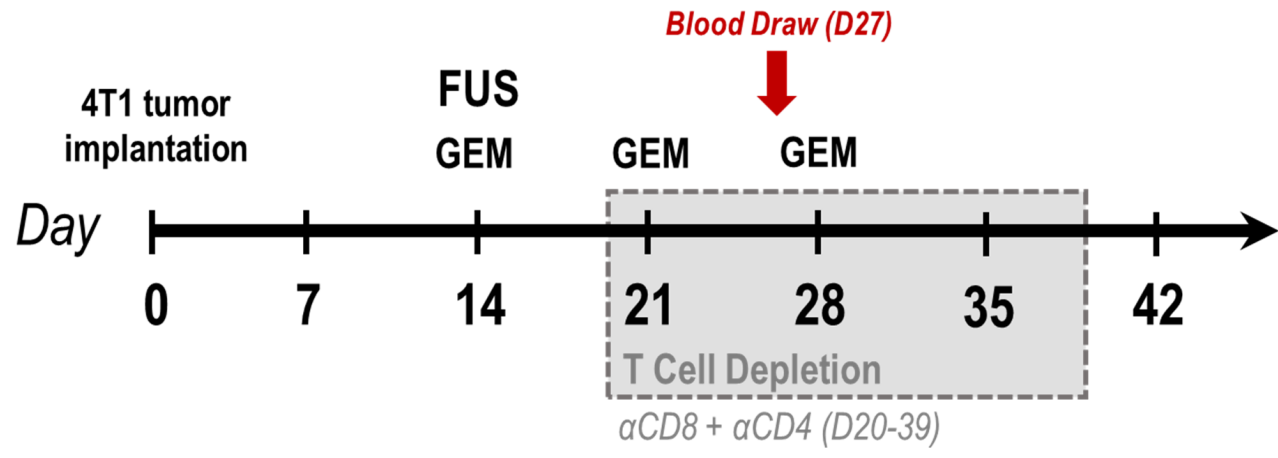

D

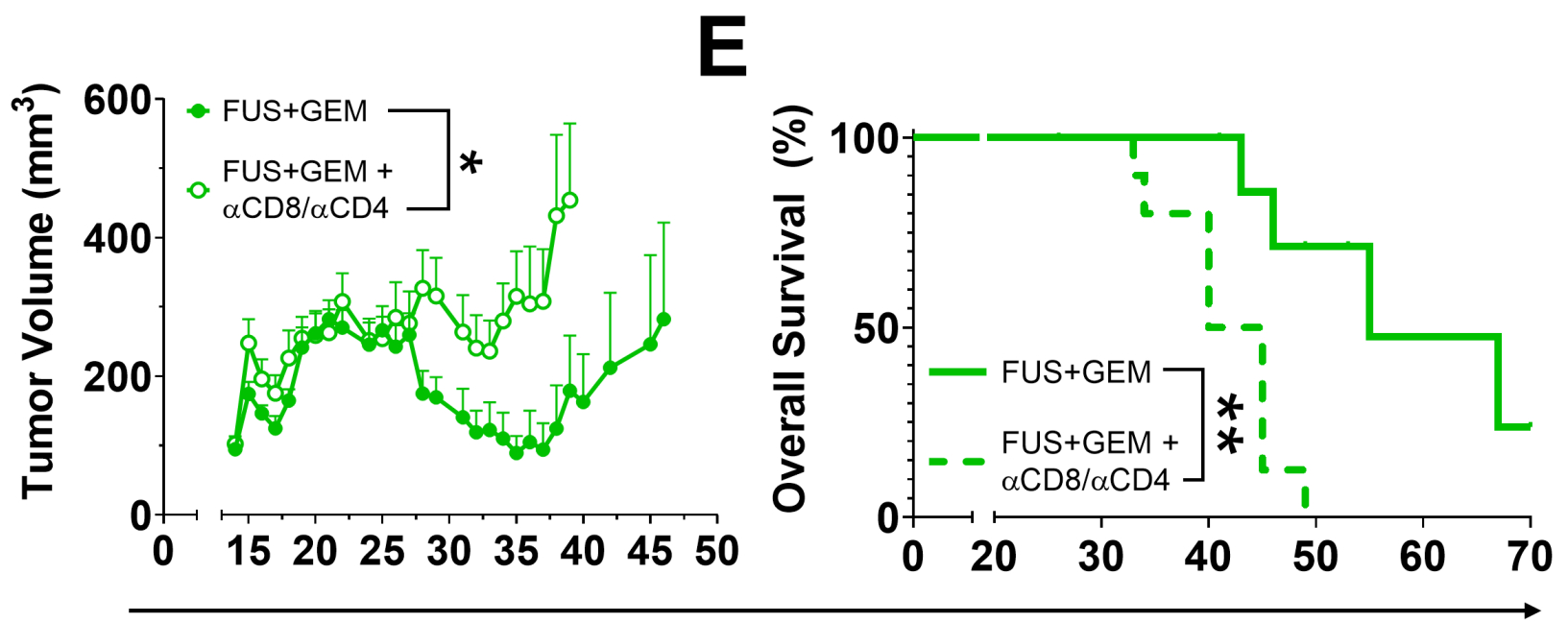

Days Post-Implantation

Figure 5 Protection conferred by combination of focusedultrasound (FUS) with gemcitabine (GEM) is dependent on adaptive immunity. (A) Average 4T1 tumor outgrowth in wild-type (WT) or Rag $1^{-/-}$mice receiving GEM monotherapy or combinatorial FUS+GEM therapy. Data are represented up to select time points corresponding with mouse dropout due to humane endpoints. $\mathrm{n}=5-7$ mice per group. Significance assessed on outgrowth up to day 37 by repeated measures mixed-effects model implementing restricted maximum likelihood method, followed by Tukey multiple comparison correction. (B) Kaplan-Meier curve depicting overall survival of Rag $1^{-/-} 4 \mathrm{~T} 1$ tumor-bearing mice receiving GEM monotherapy or combinatorial FUS+GEM therapy. $n=7-9$ per group. (C) Overview of experimental design for T cell depletions conducted on FUS+GEM background. $\alpha C D 8$ and $\alpha$ CD4 were administered on days 20, 23, 26, 29, 32, 35, and 39. On day 27, tail bleeds were performed to confirm $\mathrm{CD}^{+}$and $\mathrm{CD}^{+} \mathrm{T}$ cell depletion by flow cytometry. (D) Average 4T1 tumor outgrowth on FUS+GEM (WT) background with or without T cell depletion (' $\alpha \mathrm{CD} 8 / \alpha C D 4$ '). Data are represented up to select time points corresponding with mouse dropout due to humane endpoints. Significance assessed on outgrowth up to day 46 by repeated measures mixed-effects model implementing restricted maximum likelihood method. $n=8-9$ mice per group. (E) Kaplan-Meier curve depicting impact of $T$ cell depletion on overall survival in FUS+GEM-recipient mice (WT) bearing 4T1 tumors. $n=9-10$ mice per group. All data represented as mean \pm SEM. Significance in $(B, E)$ assessed by log-rank (Mantel-Cox) test. 'n.s.'=not significant. *p<0.05 vs indicated group(s). ${ }^{\star *} \mathrm{p}=0.0022$ vs FUS+GEM. 


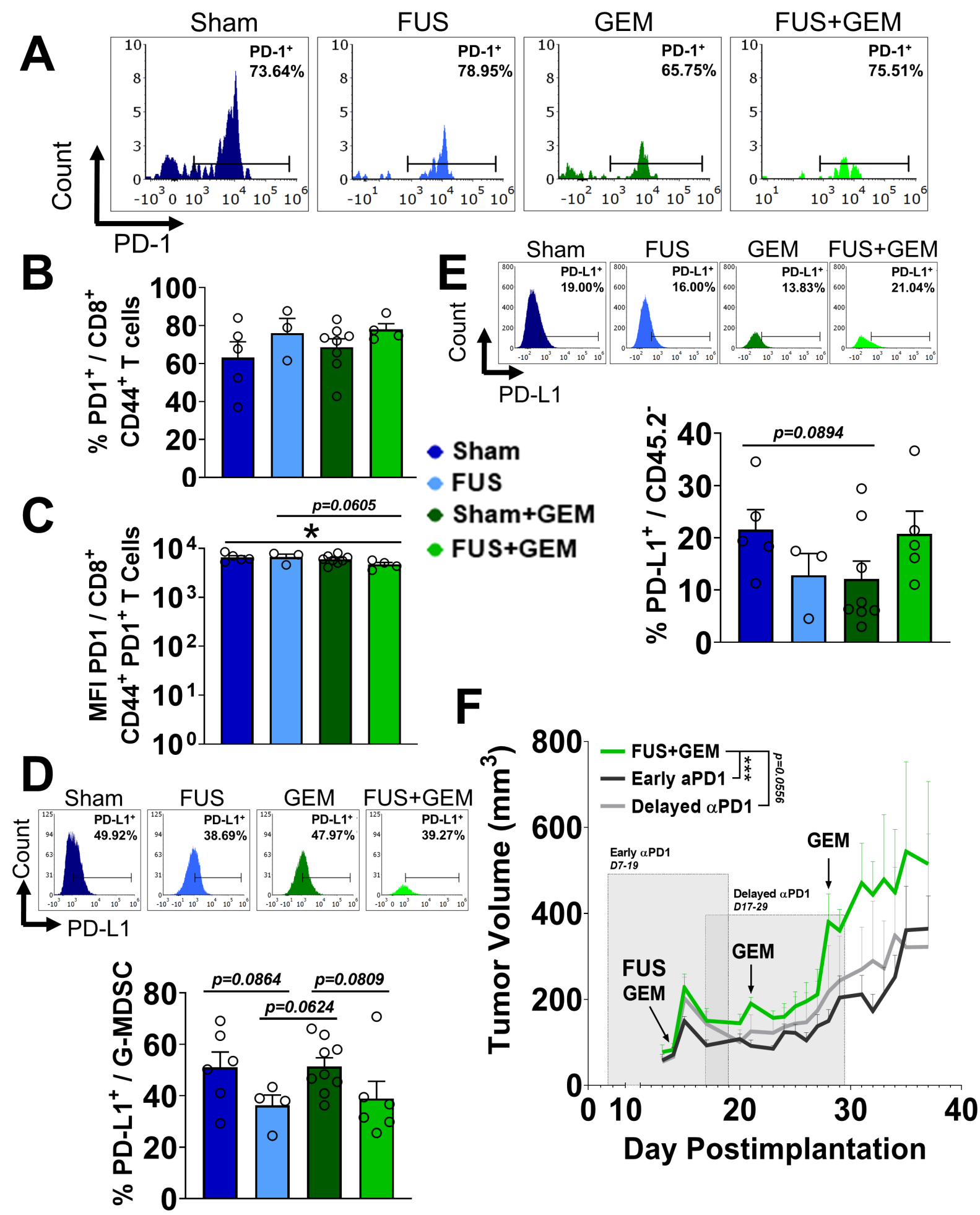

Figure 6 PD-1 blockade therapy moderately improves growth restriction conferred by FUS+GEM. (A) Representative histograms for intratumoral PD-1 expression on $\mathrm{CD}^{+} \mathrm{CD} 44^{+} \mathrm{T}$ cells across experimental groups. (B-C) Percentage of PD-1 expression (B) and PD-1 mean fluorescence intensity (C) on intratumoral CD8 ${ }^{+} \mathrm{CD} 44^{+} \mathrm{T}$ cells at 31 days postimplantation. (D-E) Percentage of PD-L1 expression on granulocytic myeloid-derived suppressor cells (G-MDSCs) (D) or CD45.2 (nonimmune) tumor/stromal cells (E) in 4T1 tumors at 31 days postimplantation with representative histograms. (F) Mice on a FUS+GEM background received $\alpha \mathrm{PD}-1$ every 3 days in either an 'early' (day 7-19) or 'delayed' (day 17-29) sequence. 4T1 tumor outgrowth in mice receiving FUS+GEM $(n=8)$, early $\alpha P D-1(n=5)$ or delayed $\alpha P D-1(n=5)$. All data represented as mean \pm SEM. Data in $(B-E)$ representative of sham $(n=5-6)$, FUS monotherapy $(n=3-4)$, GEM monotherapy $(n=8-9)$, and combinatorial FUS+GEM therapy $(n=4-6)$ groups. Significance in $(B-E)$ assessed by analysis of variance followed by Fisher's least significant difference without multiple comparisons correction for all panels. Significance in $(F)$ assessed on outgrowth up to day 37 by repeated measures mixed-effects model implementing restricted maximum likelihood method, followed by Tukey multiple comparison correction. ${ }^{*} p<0.05$ vs indicated group. ${ }^{* \star \star} p=0.0003$ vs FUS+GEM. 
decrease in PD-1 MFI resulting from FUS+GEM, neither percentage nor MFI were significantly altered by FUS or GEM therapy. At the same time point, we also evaluated PD-L1 expression on G-MDSCs (figure 6D) and nonimmune $\left(\mathrm{CD} 45.2^{-}\right)$tumor/stromal cells (figure $6 \mathrm{E}$ ) in $4 \mathrm{~T} 1$ tumors. Following application of FUS (with or without GEM), intratumoral G-MDSCs displayed a trend toward decreased PD-L1 expression. Interestingly, this trend was not recapitulated on CD45.2- cells; we noted a decreasing trend in PD-L1 expression in nonimmune cells following GEM monotherapy. Taken together, these observations generate supporting evidence for relevance of the PD-1/ PD-L1 axis as a route for tumor immune escape.

This premise led us to design a study concomitantly evaluating two driving hypotheses: (1) by intervening at the PD-1/PD-L1 axis (via PD-1 blockade), yet another mode of immunosuppression may be dampened in mice already receiving benefit from FUS+GEM, thereby giving way to more durable immunological protection; (2) timing of anti-PD-1 relative to the FUS+GEM background is critical to mounting a meaningful response against 4T1 tumors. Due to the extensive presence of MDSC, PD-1 blockade was not expected to offer benefit in the absence of additional therapy, and we first confirmed this by administering $\alpha \mathrm{PD}-1$ monotherapy (200 $\mu \mathrm{g}$ per mouse; five doses; IP) to 4T1 tumor-bearing mice. Indeed, no significant differences in tumor outgrowth were observed between treated and control mice (online supplementary figure 8 ). We next sequenced the delivery of $\alpha$ PD-1 in 4T1 tumor-bearing mice on a FUS+GEM background. Sequences were divided into an 'early' (ie, neoadjuvant priming; prior to initiation of FUS+GEM) and 'delayed' (in the adjuvant setting; within the innate immune response window acutely following initiation of FUS+GEM) sequence. 'Early' $\alpha \mathrm{PD}-1$ generated moderate, yet significant, growth constriction over FUS+GEM background (figure 6F). 'Delayed' $\alpha \mathrm{PD}-1$ trended in a similar manner to 'early' $\alpha \mathrm{PD}-1$ in its benefit beyond FUS+GEM.

\section{DISCUSSION}

The objective of this study was to test whether a clinically relevant, noninvasive therapeutic paradigm combining FUS partial thermal ablation with GEM exerts adaptive immune control in a highly aggressive model of murine metastatic mammary carcinoma. GEM was explicitly chosen on the basis of its favorable toxicity profile and ongoing investigation as a salvage single agent or as a constituent of combinatorial strategies for advanced or metastatic BrCa therapy. ${ }^{32-37}$ Our results indicate that, indeed, tumor growth control and animal survival can be markedly improved by combining FUS partial thermal ablation with GEM administration. Importantly, tumor growth inhibition correlated with increased circulating antigen-experienced $\mathrm{T}$ cells. Key experiments performed on the Rag1 $1^{-/}$background and in the setting of $\mathrm{T}$ cell depletion definitively established that tumor growth control and improved survival were entirely dependent on adaptive immunity and more specifically, $\mathrm{CD}^{+}$and $\mathrm{CD}^{+} \mathrm{T}$ cells. Taken together, our results provide strong support for the rapid translation of FUS+GEM combination therapies to clinical trials for women with metastatic TNBC.

Across both FUS systems used in this study, we performed a 'sparse' scan ablation regimen that recapitulates clinical ablation protocols set forth for ongoing clinical trials combining FUS with immunotherapy. The impact of ablation was evidenced by gross edema acutely following FUS. This observation is indicative of tissue injury and suggests the activation of key innate immune mechanisms downstream of FUS, as described in other studies. ${ }^{20-23}$ Consistent with this notion, we observed accumulation of activated DC in the tumor DLN, which supports the capacity of FUS to mobilize DC migration to the site of T cell priming. Given that mobilization of DCs in response to FUS did not translate to changes in local or peripheral T cell populations, we conjectured that MDSC-driven immunosuppression may be impeding adaptive immune response to FUS and targeted this barrier with GEM. However, additional factors may have underscored the modest immunogenicity of FUS monotherapy observed herein. Thermal ablation has the potential to denature tumor-associated antigens and disrupt vascular networks critical for the extravasation of primed T cells into tumors. Future investigations will consider whether these factors further contributed to the lack of $\mathrm{T}$ cell response to FUS.

It is noteworthy that tumor control following combinatorial therapy was induced with only a single FUS treatment at the primary tumor site, implicating the antigen/ DC/T cell axis as a likely mechanism of tumor control. Further experimentation will be needed to investigate a role for FUS in promoting tumor antigen availability and the release of inflammatory mediators that activate DC, particularly in the context of GEM co-treatment.

There is some conflict in the literature as to whether thermal ablation is sufficient to promote the immunogenicity of tumors. In a transplantable immunogenic MC38 colorectal preclinical model, sparse-scan thermal ablation monotherapy was sufficient to promote antitumor immunity. ${ }^{31}$ Further, treatment of patients with $\mathrm{BrCa}$ with thermal ablative FUS results in increased immune cell infiltration. ${ }^{28}{ }^{29}$ In contrast, recent studies have suggested that immune priming protocols involving intratumoral $\mathrm{CpG}$ and anti-PD-1 are needed to unleash the immunogenicity of thermally ablated BrCa. ${ }^{23}$ Further, intratumoral $\mathrm{CpG}$ treatment supports the immunogenicity of hyperthermia-treated BrCa. ${ }^{30}$ The argument for immune priming is based on the assertion that (1) inflammatory insults induced by FUS may result in rebound immune suppression by MDSCs and M2 polarization or (2) the degree of myeloid cell activation achieved by the release of DAMPS with thermal ablation-induced sterile inflammation is insufficient. Our data support the latter argument, as modest intratumoral DC activation was observed with ablative FUS alone, with negligible impact on MDSC. 
While application of FUS to well-established 4T1 tumors modestly impacted 4T1 tumor outgrowth, significantly greater efficacy was observed when GEM was introduced to this treatment regimen. The concomitant use of FUS and GEM was sufficient to significantly restrict primary tumor outgrowth in 4T1 and E0771 models of metastatic TNBC on different mouse genetic backgrounds. We additionally recapitulated this finding in the 4T1 model using the research-grade analog of a clinically relevant FUS system. Notably, despite consistency in FUS and GEM treatment protocols across metastatic TNBC models, growth constraint was most striking in the 4T1 model-perhaps owing to its dependency on G-MDSCs. The reduced susceptibility to GEM and dampened impact of FUS+GEM in E0771 may be attributed to its distinct immunological profile; in contrast with 4T1, immunosuppression in the E0771 model is driven by an abundance of M-MDSC (ie, highly enriched Ly6C expression on myeloid cells), M2 macrophages and regulatory $\mathrm{T}$ cells (Dr Melanie Rutkowski, personal communication, 2020), with very low frequency of G-MDSC. ${ }^{38-40}$ One further potential explanation might be offered by recent evidence that multiple doses of GEM administration in E0771 can enhance the immunosuppressive activity of M-MDSC despite modestly impeding tumor outgrowth. ${ }^{40}$

It is also possible that the elevated antitumor immunity resulting from FUS and GEM co-treatment is due to an ability of FUS to sensitize the tumor to GEM activity or access, thereby decreasing immunosuppressive networks. We do not favor this argument as the half-life of GEM is very short in mice ${ }^{41}$ and the timing of its administration relative to FUS in our paradigm is inconsistent with this explanation. Rather, our Rag $1^{-/-}$findings indicate that the adaptive immune compartment plays a critical role in the protection offered by FUS+GEM, and our T cell depletion findings meanwhile suggest that $\mathrm{CD} 8^{+}$and $\mathrm{CD}^{+} \mathrm{T}$ cell are key mediators of this protection. While these findings are corroborated by a marked increase in circulating activated $\mathrm{T}$ cells, we do not observe corresponding increases in $\mathrm{T}$ cell numbers or polyfunctionality within the intratumoral lymphocyte pool. Interestingly, FUS+GEM elevates the percentage of NK, T and B cells in the spleens of 4T1 tumor-bearing mice relative to sham and monotherapy groups. Future studies warrant evaluating the contribution of these cell types to the protective effects conferred by FUS+GEM. We selected time points for immune characterization of $4 \mathrm{~T} 1$ tumors with the intent to capture the early stages of FUS-induced de novo intratumoral $\mathrm{T}$ cell response (day 7; figure 1) in addition to the immune-dependent sustained protection resulting from intervention with FUS and/or GEM (day 31; figures 3-4); however, it will be imperative to evaluate additional time points in future studies to more deeply resolve the dynamics of innate and adaptive immunity following these interventions.

We also determined that the impact of FUS+GEM on the primary tumor translated to extension of overall survival of $4 \mathrm{~T} 1$ tumor-bearing mice. The survival of mice in this study was exclusively based on humane endpoints relating to onset of pulmonary metastatic burden (as opposed to primary tumor volume). There are two possible hypotheses to explain the reduced mortality. First, the ability of FUS+GEM to limit primary tumor growth may delay metastatic seeding in the lungs. Alternatively, increased systemic antitumor immunity driven by FUS+GEM could limit the growth of metastatic lesions. This is supported by our observation of increased circulating $\mathrm{CD}^{+}$and $\mathrm{CD}^{+} \mathrm{T}$ cells, as well as the increased proportion of these $\mathrm{T}$ cells expressing CD44 (indicating antigen experience). Since we utilized overall survival as a proxy for metastatic burden, future studies to this end may warrant resection of the primary tumor in order to generate a reasonable window assessing pulmonary metastatic burden across therapies.

Our results are consistent with the hypothesis that GEM can confer the same effect as other systemically administered immunoadjuvants without the need for direct injection or priming. Specifically, in our study, the effects of $\mathrm{CpG}$ can be replicated by attenuating myeloid cell activity through the use of GEM concomitantly initiated with FUS-offering the advantage of systemic and noninvasive administration compared with intratumoral $\mathrm{CpG}$ delivery. However, it should be noted that while GEM treatment leads to G-MDSC knockdown, this effect is neither complete nor durable. Thus, while the efficacy of FUS+GEM is encouraging, tumors escaped control once GEM administration was terminated in combinationtreated mice. Thus, immunosuppression may be rapidly re-established, which may explain the limited difference in intratumoral $\mathrm{T}$ cell function observed among groups, as well as elevated TNF $\alpha$ production by G-MDSC in GEMrecipient groups.

It is also possible that alternative mechanisms of immunosuppression are compensating for MDSC inhibition by GEM or are additive with MDSC rebound following GEM. This could in part explain the lack of complete or durable tumor control with FUS+GEM. For example, we observed a modest increase in percentage of Tregs in the spleens of mice treated with FUS+GEM. Although Tregs constitute a low fraction of cells in the spleen, their increase in the context of combinatorial therapy is noteworthy as they could functionally contribute to the lack of durable tumor constriction by compensating for reduction in MDSC-driven immunosuppression. Deciphering the resistance mechanisms that are activated by thermally ablative FUS is necessary for its successful implementation as a therapeutic modality in BrCa.

In the future, it will be important to consider whether alternative strategies that lead to more robust, durable limitation of G-MDSC-mediated immunosuppression are more effective than GEM. Such strategies could include elimination (eg, $\alpha$ GR-1 or 5-FU), trafficking impairment (eg, chemokine blockade) and myeloid (re-)polarization (eg, TLR agonists and agents that promote functional alterations). That said, enthusiasm for GEM is heightened relative to several of these therapies due to 
its clinical accessibility and relevance to standard $\mathrm{BrCa}$ treatment.

These findings warrant further exploration of GEM timing (ie, administration before vs after FUS), GEM dosing or route of administration (ie, direct intratumoral injection) as well as repeated FUS treatments. Moreover, our utilization of a sparse-scanning strategy designed to achieve a low-yet meaningful-tumor volume ablation fraction may have limited the degree of overall tissue insult, which could in turn evoke mechanisms of canonical wound healing aligned with immunosuppression in the TME. Future studies ought to consider alternative thermal ablation strategies (eg, higher ablation fractions) in combination with GEM. Growing evidence in the literature also points to mechanical FUS regimes being immunologically favorable. ${ }^{42} 43$ GEM may serve favorably as an adjunct to mechanical ablation techniques (eg, histotripsy) as well.

Interestingly, we observed some modest activity of PD-1 blockade in combination with FUS+GEM treatment. If we here consider FUS as an 'auto-vaccination' strategy, studies suggest that the sequencing of anti-PD-1 relative to vaccination or other proper forms of priming is indeed crucial. ${ }^{44}$ The concomitant administration of adjuvants such as GEM may serve as one strategy to curb suppressive mechanisms that would ultimately dampen the priming effect of FUS. Our findings show that initiating $\alpha \mathrm{PD}-1$ therapy prior to versus shortly after FUS+GEM does not bear a marked difference on 4T1 primary tumor outgrowth. The limited impact of PD-1 blockade on this background may be explained in part by the observation that a major source of PD-L1 in the TME is MDSC (a cell population already being inhibited by GEM), and the likelihood that other mechanisms of resistance to tumor immunity may operate in the current model system. While we here interrogated the role of PD-1 blockade in the setting of combinatory FUS+GEM therapy, future studies could also consider targeting PD-L1 or alternative immune checkpoints such as Tim3, CTLA-4 or OX-40.

That said, these observations in the context of PD-1 blockade are timely, as a clinical trial investigating the role of pembrolizumab sequencing relative to FUS partial thermal ablation (using the Theraclion Echopulse System) in patients with recurrent $\mathrm{BrCa}$ is currently underway at the University of Virginia. Our study generates important insights for this and future clinical evaluations of FUS and immunotherapy.

\section{CONCLUSION}

We herein describe a novel, readily translatable paradigm for combination of FUS partial thermal ablation with a clinically approved, myeloreductive chemotherapy (GEM). We demonstrate that FUS+GEM constrains primary tumor outgrowth and improves survival in murine TNBC. Moreover, we unveil a critical role for adaptive immunity in the efficacy of this combination. These findings generate timely and provocative insights into the immunogenicity of FUS thermal ablation and the role of GEM as adjunct to this approach.

Twitter Natasha D Sheybani @ndsheybani and Richard J Price @PriceLabUVaBME

Acknowledgements We thank the UVA Carter Immunology Center for enabling generation of flow cytometry data. We thank Aaron Stevens for technical assistance on flow cytometry studies and Dr Hideo Yagita (Jutendo University) for providing $\alpha \mathrm{PD}-1$ antibody. We also thank Dr Anil Shanker (Meharry Medical College) and Dr Drew Dudley (UVA) for providing 4T1 and E0771 cell lines, respectively. We are also grateful to Dr Patrick Dillon (UVA) and Dr David Brenin (UVA) for offering feedback and clinical perspectives throughout the development of this work.

Contributors NDS and ARW designed and performed experiments, analyzed the data, and wrote the manuscript. EAT provided technical support for FUS studies. HY provided reagents. TNJB and RJP provided funding support, supervised all studies, and contributed to writing the manuscript. All authors reviewed and approved the manuscript.

Funding This study was supported by National Institutes of Health Grants R01CA197111 (RJP and TNJB) and R21CA230088 (RJP), the Focused Ultrasound Foundation (TNJB and RJP), and Theraclion (TNJB and RJP). NDS was supported by a National Cancer Institute F99/K00 Predoctoral to Postdoctoral Fellow Transition Award (F99CA234954), NSF Graduate Research Fellowship, and UVA School of Medicine Wagner Fellowship. ARW was supported by an NIH Cancer Training Grant (2T32CA009109-41) the UVA Cancer Center Farrow Fellowship and NCI Cancer Center Support Grant P30 CA44579.

Competing interests None declared.

Patient consent for publication Not required.

Ethics approval The murine experiments were prospectively reviewed and approved by the University of Virginia Animal Care and Use Committee.

Provenance and peer review Not commissioned; externally peer reviewed.

Data availability statement All data relevant to the study are included in the article or uploaded as supplementary information. N/A.

Open access This is an open access article distributed in accordance with the Creative Commons Attribution Non Commercial (CC BY-NC 4.0) license, which permits others to distribute, remix, adapt, build upon this work non-commercially, and license their derivative works on different terms, provided the original work is properly cited, appropriate credit is given, any changes made indicated, and the use is non-commercial. See http://creativecommons.org/licenses/by-nc/4.0/.

\section{ORCID iDs}

Timothy N J Bullock http://orcid.org/0000-0001-6141-3261

Richard J Price http://orcid.org/0000-0002-0237-2102

\section{REFERENCES}

1 Miyashita M, Sasano H, Tamaki K, et al. Prognostic significance of tumor-infiltrating CD8+ and Foxp3+ lymphocytes in residual tumors and alterations in these parameters after neoadjuvant chemotherapy in triple-negative breast cancer: a retrospective multicenter study. Breast Cancer Res 2015;17:124.

2 Nanda R, Chow LQM, Dees EC, et al. Pembrolizumab in patients with advanced triple-negative breast cancer: phase Ib KEYNOTE-012 study. J Clin Oncol 2016;34:2460-7.

3 Emens LA, Cruz C, Eder JP, et al. Long-Term clinical outcomes and biomarker analyses of Atezolizumab therapy for patients with metastatic triple-negative breast cancer: a phase 1 study. JAMA Oncol 2019;5:74-82.

4 Adams S, Schmid P, Rugo HS, et al. Pembrolizumab monotherapy for previously treated metastatic triple-negative breast cancer: cohort a of the phase II KEYNOTE-086 study. Ann Oncol 2019;30:397-404.

5 Voorwerk L, Slagter M, Horlings HM, et al. Immune induction strategies in metastatic triple-negative breast cancer to enhance the sensitivity to PD-1 blockade: the tonic trial. Nat Med 2019;25:920-8.

6 Gabrilovich DI, Ostrand-Rosenberg S, Bronte V. Coordinated regulation of myeloid cells by tumours. Nat Rev Immunol 2012;12:253-68.

7 Gabrilovich DI, Nagaraj S. Myeloid-Derived suppressor cells as regulators of the immune system. Nat Rev Immunol 2009;9:162-74.

8 Diaz-Montero CM, Salem ML, Nishimura MI, et al. Increased circulating myeloid-derived suppressor cells correlate with clinical cancer stage, metastatic tumor burden, and doxorubicin- 
cyclophosphamide chemotherapy. Cancer Immunol Immunother 2009:58:49-59.

9 Vonderheide RH, Glennie MJ. Agonistic CD40 antibodies and cancer therapy. Clin Cancer Res 2013:19:1035 LP-43.

10 Suzuki E, Kapoor V, Jassar AS, et al. Gemcitabine selectively eliminates splenic Gr-1+/CD11b+ myeloid suppressor cells in tumor-bearing animals and enhances antitumor immune activity. Clin Cancer Res 2005;11:6713-21.

11 Vincent J, Mignot G, Chalmin F, et al. 5-Fluorouracil selectively kills tumor-associated myeloid-derived suppressor cells resulting in enhanced T Cell-Dependent antitumor immunity. Cancer Res 2010;8:3690.

12 Le HK, Graham L, Cha E, et al. Gemcitabine directly inhibits myeloid derived suppressor cells in BALB/c mice bearing $4 \mathrm{~T} 1$ mammary carcinoma and augments expansion of $\mathrm{T}$ cells from tumor-bearing mice. Int Immunopharmacol 2009;9:900-9.

13 Curley CT, Sheybani ND, Bullock TN, et al. Focused ultrasound immunotherapy for central nervous system pathologies: challenges and opportunities. Theranostics 2017;7:3608-23.

14 Sheybani ND, Price RJ. Perspectives on recent progress in focused ultrasound immunotherapy. Theranostics 2019;9:7749-58.

15 Yuan S-M, Li H, Yang M, et al. High intensity focused ultrasound enhances anti-tumor immunity by inhibiting the negative regulatory effect of miR-134 on CD86 in a murine melanoma model. Oncotarget 2015;6:37626-37.

16 Wang X, Sun J. High-Intensity focused ultrasound in patients with late-stage pancreatic carcinoma. Chin Med J 2002;115:1332-5.

17 Madersbacher S, Gröbl M, Kramer G, et al. Regulation of heat shock protein 27 expression of prostatic cells in response to heat treatment. Prostate 1998;37:174-81.

18 Kramer G, Steiner GE, Gröbl M, et al. Response to sublethal heat treatment of prostatic tumor cells and of prostatic tumor infiltrating T-cells. Prostate 2004;58:109-20.

19 Huang X, Yuan F, Liang M, et al. M-HIFU inhibits tumor growth, suppresses STAT3 activity and enhances tumor specific immunity in a transplant tumor model of prostate cancer. PLoS One 2012;7:e41632.

$20 \mathrm{Hu}$ Z, Yang XY, Liu Y, et al. Investigation of HIFU-induced anti-tumor immunity in a murine tumor model. $J$ Transl Med 2007;5:34.

21 Wu F, Wang Z-B, Lu P, et al. Activated anti-tumor immunity in cancer patients after high intensity focused ultrasound ablation. Ultrasound Med Biol 2004;30:1217-22.

22 Deng J, Zhang Y, Feng J, et al. Dendritic cells loaded with ultrasound-ablated tumour induce in vivo specific antitumour immune responses. Ultrasound Med Biol 2010;36:441-8.

23 Silvestrini MT, Ingham ES, Mahakian LM, et al. Priming is key to effective incorporation of image-guided thermal ablation into immunotherapy protocols. JCl Insight 2017;2:e90521.

24 Hundt W, O'Connell-Rodwell CE, Bednarski MD, et al. In vitro effect of focused ultrasound or thermal stress on Hsp70 expression and cell viability in three tumor cell lines. Acad Radiol 2007:14:859-70.

25 Kruse DE, Mackanos MA, O'Connell-Rodwell CE, et al. Shortduration-focused ultrasound stimulation of Hsp70 expression in vivo. Phys Med Biol 2008;53:3641-60.

26 Wu F, Wang Z-B, Cao Y-D, et al. Expression of tumor antigens and heat-shock protein 70 in breast cancer cells after high-intensity focused ultrasound ablation. Ann Surg Oncol 2007;14:1237-42.
27 Hu Z, Yang XY, Liu Y, et al. Release of endogenous danger signals from HIFU-treated tumor cells and their stimulatory effects on APCS. Biochem Biophys Res Commun 2005;335:124-31.

28 Xu Z-L, Zhu X-Q, Lu P, et al. Activation of tumor-infiltrating antigen presenting cells by high intensity focused ultrasound ablation of human breast cancer. Ultrasound Med Biol 2009;35:50-7.

29 Lu P, Zhu X-Q, Xu Z-L, et al. Increased infiltration of activated tumor-infiltrating lymphocytes after high intensity focused ultrasound ablation of human breast cancer. Surgery 2009;145:286-93.

30 Kheirolomoom A, Silvestrini MT, Ingham ES, et al. Combining activatable nanodelivery with immunotherapy in a murine breast cancer model. J Control Release 2019;303:42-54.

31 Liu F, Hu Z, Qu L, et al. Boosting high-intensity focused ultrasoundinduced anti-tumor immunity using a sparse-scan strategy that can more effectively promote dendritic cell maturation. J Transl Med 2010;8:7.

32 Albain KS, Nag SM, Calderillo-Ruiz G, et al. Gemcitabine plus paclitaxel versus paclitaxel monotherapy in patients with metastatic breast cancer and prior anthracycline treatment. $J$ Clin Oncol 2008:26:3950-7.

33 O'Shaughnessy J, Schwartzberg L, Danso MA, et al. Phase III study of iniparib plus gemcitabine and carboplatin versus gemcitabine and carboplatin in patients with metastatic triple-negative breast cancer. J Clin Oncol 2014;32:3840-7.

34 Carmichael J, Possinger K, Phillip P, et al. Advanced breast cancer: a phase II trial with gemcitabine. J Clin Oncol 1995;13:2731-6.

35 Nagourney RA, Link JS, Blitzer JB, et al. Gemcitabine plus cisplatin repeating doublet therapy in previously treated, relapsed breast cancer patients. J Clin Oncol 2000;18:2245-9.

36 Seidman AD. Gemcitabine as single-agent therapy in the management of advanced breast cancer. Oncology 2001;15:11-14.

37 Lüftner D, Flath B, Akrivakis C, et al. Gemcitabine for palliative treatment in metastatic breast cancer. $J$ Cancer Res Clin Oncol 1998:124:527-31.

38 Crosby EJ, Wei J, Yang XY, et al. Complimentary mechanisms of dual checkpoint blockade expand unique T-cell repertoires and activate adaptive anti-tumor immunity in triple-negative breast tumors. Oncoimmunology 2018;7:e1421891.

39 Yu Y-RA, O'Koren EG, Hotten DF, et al. A protocol for the comprehensive flow cytometric analysis of immune cells in normal and inflamed murine non-lymphoid tissues. PLoS One 2016:11:e0150606

40 Wu C, Tan X, Hu X, et al. Tumor Microenvironment following Gemcitabine Treatment Favors Differentiation of Immunosuppressive Ly6C high Myeloid Cells. J Immunol 2020;204:212 LP-23.

41 Shipley LA, Brown TJ, Cornpropst JD, et al. Metabolism and disposition of gemcitabine, and oncolytic deoxycytidine analog, in mice, rats, and dogs. Drug Metab Dispos 1992;20:849-55.

42 Eranki A, Srinivasan P, Ries M, et al. High-Intensity focused ultrasound (HIFU) triggers immune sensitization of refractory murine neuroblastoma to checkpoint inhibitor therapy. Clin Cancer Res 2020;26:1152-61.

43 Schade GR, Wang Y-N, D'Andrea S, et al. Boiling Histotripsy ablation of renal cell carcinoma in the Eker rat promotes a systemic inflammatory response. Ultrasound Med Biol 2019;45:137-47.

44 Verma V, Shrimali RK, Ahmad S, et al. PD-1 blockade in subprimed CD8 cells induces dysfunctional PD $-1^{+}$CD $38^{\text {hi }}$ cells and anti-PD-1 resistance. Nat Immunol 2019;20:1231-43. 